UNIVERSITÉ DU QUÉBEC

MÉMOIRE PRÉSENTÉ À

L'UNIVERSITÉ DU QUÉBEC À CHICOUTIMI

COMME EXIGENCE PARTIELLE

DE LA MAÎTRISE EN ART (M.A.)

PAR

JESSYKA MALTAIS JEAN

L'EXPÉRIENCE SENSIBLE CHEZ LE SPECTATEUR :

DE LA PHYSIOLOGIE A L'EMPATHIE.

MAI 2009 
RÉSUMÉ

Cette recherche d'ordre fondamentale tente de cerner la nature physiologique de l'expérience sensible chez le spectateur d'évènements scéniques. L'origine de ce questionnement remonte à mes propres expériences sensibles vécues lors de cet échange qu'est le théâtre, autant comme spectatrice que comme performer.

En cherchant des réponses du coté des neurosciences, je me suis questionnée pour savoir s'il se produisait un phénomène d'empathie physiologique chez le spectateur. Cette interrogation a créé un lien entre la théorie des neurones miroirs, l'empathie et l'évènement scénique.

A l'aide de l'approche systémique, l'objectif est devenu la modélisation de cet échange entre les deux systemes vivants que sont le performer et le spectateur. Les signaux, d'ordre physique ou chimique, s'avèrent être les vecteurs énergétiques de cette communication physiologique entre deux corps durant ce moment particulier.

L'évènement scénique est décrit ici en fonction de certaines conditions telles que l'organicité, l'art performatif, l'esthétique de la stimulation et la subjectivité du spectateur. Cet évènement s'inscrit d'alleurs, en premier lieu, dans le cadre des sciences qui s'intéressent au spectacle vivant comme l'ethnoscénologie. Puis, elle s'ajoute dans un courant plus large, appelé théâtre postdramatique, qui place l'humain au centre de ses préoccupations.

Le phénomène esthétique qu'est l'expérience sensible est riche et possède plusieurs niveaux de complexité. À partir des processus d'intégration de l'information sensorielle dans le corps du spectateur avec le système nerveux et endocrinien, jusqu'au concept d'empathie et de la théorie des neurones miroirs, ce mémoire veut saisir la complexité physiologique de l'expérience sensible.

EXPÉRIENCE SENSIBLE - SPECTATEUR - PERFORMER - CORPS SYSTÉMIQUE - STIMULI - ÉVĖNEMENT SCÉNIQUE - EMPATHIE 
REMERCIEMENTS

Je tiens à remercier particulièrement mon directeur de recherche, monsieur Rodrigue Villeneuve.

Merci aux membres de mon jury, messieurs Robert Faguy et Jean-Paul Quéinnec.

Merci au module des arts de l'Université du Québec à Chicoutimi, aux professeurs, chargés de cours et techniciens qui m'ont enseigné leur passion.

Je remercie également ma famille et mes amis pour leurs précieux encouragements jusqu'à ce moment.

Enfin, Yannick et Léa, les mots ne suffisent pas. Votre présence et votre énergie ont été mon support de tous les instants, sous toutes ses formes. Merci profondément. 


\section{LISTE DES FIGURES}

1. Carte des concepts...............................................vi

2. Modélisation d'un atome................................................

3. Molécule théâtrale.................................................... 10

4. Paradigme systémique selon Le Moigne.................................... 14

5. Ouverture d'un système................................................... 15

6. Niveaux hiérarchiques entre systèmes et sous-systèmes...................... 16

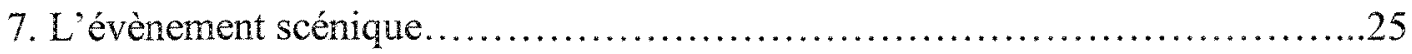

8. Le butô comme art performatif et pratique systémique........................ 33

9. Butô : Tanaka Min en Islande......................................... 34

10. Butô : Training dirigé par Murobushi $\mathrm{K}$. au Japon......................... 35

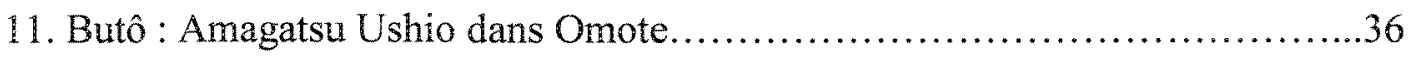

12. Stimuli à l'œuvre lors de l'évènement scénique..........................40

13. Systèmes de traitement des stimuli..................................42

14. Système endocrinien................................................. 43

15. Système nerveux................................................. 44

16. Récepteurs de la peau.............................................. 52

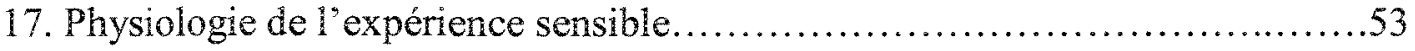

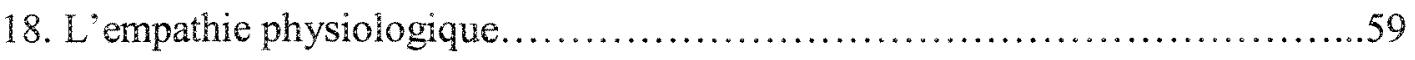

19. L'expérience sensible chez le spectateur : de la physiologie à l'empathie......67 


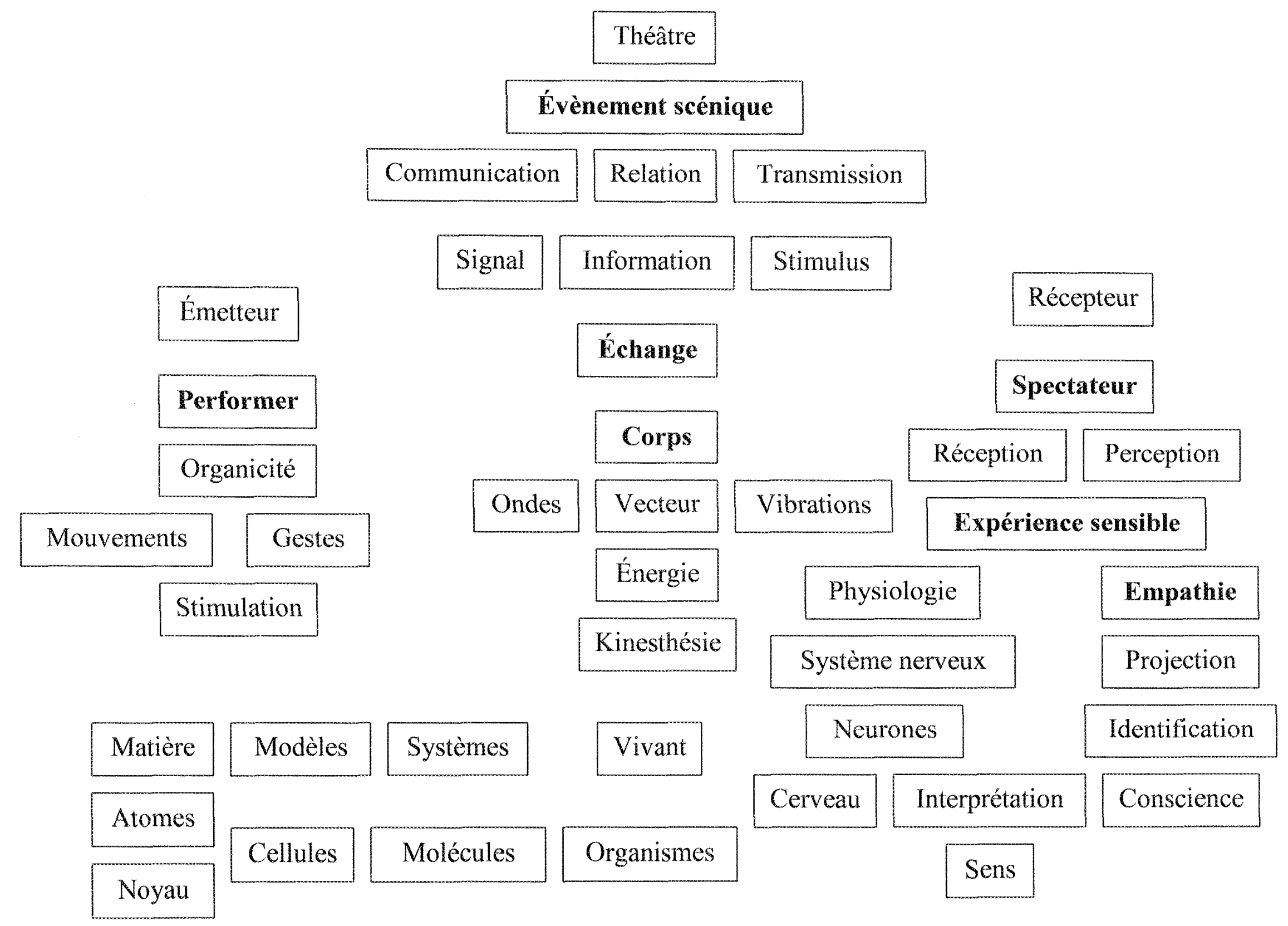




\section{TABLE DES MATIÈRES}

Résumé.......................................................... ii

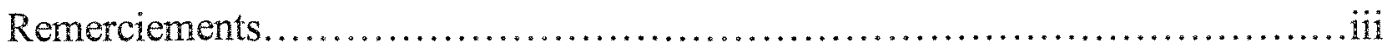

Liste des figures.......................................................

Introduction................................................................

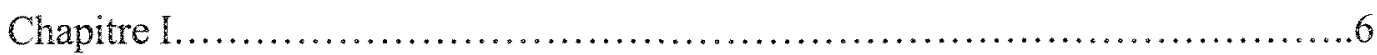

L'approche systémique: de la structure de la matière à la modélisation de l'évènement scénique

Chapitre II......................................................... 18

L'ethnoscénologie comme macrosystème théorique de l'évènement scénique

Chapitre III.............................................................. 37

L'expérience sensible chez le spectateur: de la physiologie à l'empathie

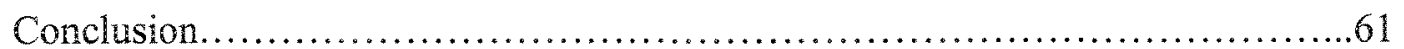

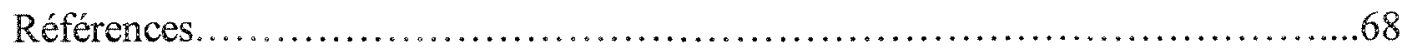


INTRODUCTION 
Quand vous êtes assis au théâtre et que plus personne ne dit mot sur la scène, quelque chose vous parle encore, s'adresse à votre être profondément physique : le langage organique, la seule présence de cet autre corps devant vous. Un échange tacite s'installe : l'organisme spectateur devient percepteur dans le matériau qu'est sa chair.

Cette recherche émerge de mes propres expériences sensibles vécues lors d'évènements scéniques, autant comme spectatrice que comme performer'. Ce terme n'est d'ailleurs pas employé inutilement et sera défini plus avant dans le contexte de cette recherche. En effet, il m'est arrivé quelques fois, d'une façon extraordinaire, d'être littéralement «frappée » par une expérience sensible lors d'une représentation. Ces rares moments d'une incroyable puissance physiologique, quasi métaphysique, demeurent des influents majeurs de ma vie. J'ai toujours cherché à comprendre comment ce phénomène esthétique, cette expérience physiologique se produit, et pourquoi ne survient-elle pas à chaque fois que l'on se présente au théâtre.

Ayant longtemps cheminé en sciences, plus particulièrement en biologie, je suis fascinée par le corps humain, par sa riche complexité. Cette recherche et ce qui anime

\footnotetext{
'Pavis déninit le performer dans son dictionnaire de thêâtre comme quelqu'un «qui réalise toujours un exploit (une performance) vocal, gestuel, ou instrumental, par opposition à l'interprétation et la représentation mimétique du rôle par l'acteur.» Thomas Richard ajoute (dans Leao p.47) «que le performer est un restaurateur du comportement organique».
} 
ma propre démarche artistique résultent donc du métissage de ces deux disciplines. Par conséquent, mes préoccupations théâtrales se situent au niveau biologique et physiologique. Il y est question de la vie, mais surtout du corps, matrice fondamentale et systémique d'où émane cette vie.

Le théâtre, la salle de spectacle, le lieu, l'espace-temps deviennent ici un laboratoire où deux organismes vivants, deux systèmes ouverts ef dynamiques sont mis en présence dans un contexte particulier: l'évènement scénique. L'échange entre le performer et le spectateur est certain; mais comment le définir, le comprendre physiologiquement dans un contexte artistique? Que se passe-t-1l chez le spectateur lors de l'évènement scénique? L'expérience sensible est-elle liée à l'organicité du performer et/ou de 1'évènement scénique?

Dans la suite de mes préoccupations physiologiques, j'ai tenté de trouver des réponses du coté des neurosciences et c'est alors que je me suis également interrogée sur la possibilité qu'il se produise un phénomène d'empathie physiologique chez le spectateur lors de l'évènement scénique. En creusant davantage cette piste, un parallèle s'est rapidement tissé entre l'empathie physiologique et ce vieux concept de catharsis chez Aristote.

J'aborde le monde avec la conviction de l'unité et de l'interdépendance de tous les phénomènes. J'ai le profond sentiment que tout ce qui nous entoure est relié : 
«L'univers entier apparaît comme un réseau dynamique de structures énergétiques interdépendantes.» ${ }^{2}$. La construction de cette recherche, comme un reflet de ces convictions, s'immisce au creux des sillons de l'approche systémique: des liens, des réseaux et des modèles, une circulation, des flux d'informations, de l'énergie dynamique entre les éléments. Je cherche, ici, à saisir la complexité de l'expérience sensible en ayant une vision globale de cette dernière. Ma compréhension passe par la représentation de ce phénomène.

Dans cette perspective d'une recherche plus fondamentale, davantage axée sur la théorie que sur la création, l'objectif est devenu la modélisation des différents « objets » du système théâtral qui entrent en relation lors de l'évènement scénique. Cela me permettra de comprendre, de définir l'expérience sensible et, peut-être, trouver réponse à mon questionnement: est-il possible qu'il se produise un phénomène d'empathie physiologique chez le spectateur lors de l'évènement scénique?

À cette fin, le premier chapitre sera consacré à l'approche systémique, qui représente l'angle méthodologique privilégiée ici, tout en traduisant aussi ma façon de voir le monde, d'appréhender le réel. Son utilité, sa définition ainsi que quelques aspects techniques concernant cette approche seront élaborés afin d'éclaircir sa présence dans les pages suivantes.

${ }^{2}$ Capra, F. (1975). Le tao de la physique. Paris : Editions Sand. Page 53 
Le second chapitre présentera le cadre théorique, le macrosystème dans lequel s'inscrit mon questionnement. Il sera constitué tout d'abord d'une introduction à l'ethnoscénologie, la discipline qui étudie les spectacles vivants. Suivront ensuite les concepts-clé qui défnissent l'évènement scénique ainsi que les éléments qui en découlent, comme balises de cette recherche. Enfin, je me permettrai une brève incursion dans le thêâtre postdramatique, courant de pensée auquel, il me semble, cet essai appartient.

En demier lieu, le troisième chapitre, qui constitue le noyau de ma réflexion, abordera l'aspect physiologique de l'expérience sensible chez le spectateur. C'est-à-dire les signaux, qui agissent comme autant de stimuli et qui sont les vecteurs de l'expérience sensible, ensuite les systèmes de traitement et le processus d'intégration de cette information sensorielle dans le corps du spectateur. Suivra l'explication fournie par le concept d'empathie physiologique et la théorie des neurones miroir. Cet aspect neurologique apporte un éclairage très porteur sur l'expérience sensible.

Une certitude s'installe: le corps, dans sa matérialité, est à la base de cette recherche. C'est-à partir de cette organicité systémique que seront explorées les avenues qui sous-tendent mes intuitions et ma compréhension de cet art de relation qu'est le theâtre; cerner la nature et les fondements physiologiques de l'expérience sensible dans le corps du spectateur, lors de l'évènement scénique dans son ensemble, mais surtout, lors de la mise en présence d'un autre système vivant: le performer. 


\section{CHAPITRE I}

L'approche systémique : de la structure de la matière à la modélisation du théatre 
Mon unique saisie sur le monde se fait à partir de mon seul corps, de mes sens constamment stimulés par toutes les formes d'énergie environnantes. Je baigne physiologiquement dans une multitude de vibrations, de longueurs d'onde qui composent la matérialité de ce que je suis et de ce qui m'entoure. Mon corps, lui-même un champ vibrant, s'harmonise et s'ajuste au diapason de cette danse moléculaire

Je suis un système autonome. Mais je suis liée, interdépendante, construite à partir de centaine d'antres systèmes en étroite collaboration.

Tel qu'établit en introduction, ce chapitre présente l'angle méthodologique de cette recherche, c'est-à-dire l'approche systémique. Cette discipline, relativement nouvelle, correspond à la façon dont je comprends le monde qui m'entoure et dont je suis moi-même partie prenante. Voilà donc pourquoi le théâtre est traité, ici, sous forme systémique et pourquoi autant d'importance est accordée à cet aspect.

Le lecteur sera d'abord initié à ma conception, quelque peu fantasmatique, de la molécule théâtrale. Viendront ensuite les grands principes théoriques de l'approche systémique me permettant de modéliser, donc de saisir dans sa globalité, l'expérience sensible. 


\section{La molécule théâtrale}

Largement défini par les sciences fondamentales, l'univers entier n'est qu'une composition de multiples systèmes à différentes échelles. Ce concept de système est à la base même de la vie qui s'amorce à partir de la matière organique, elle-même composée du plus petit système identifiable: l'atome. Ce dernier est le constituant physique élémentaire de la matière, un assemblage de particules fondamentales.

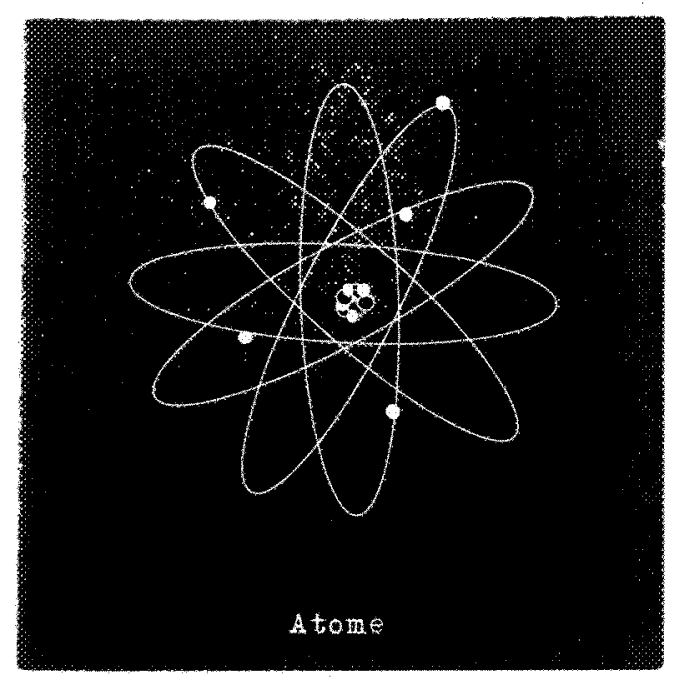

Figure 2. Modélisation d'un atome

La réalité m'apparait avant tout comme l'oscillation vibratoire d'atomes. Mes mains, ce clavier où elles se posent, la chaise me supportant représentent diverses formes de cette réalité. La vie découle de la combinaison spécifique de ces atomes entre eux: d'abord il y a les molécules, ensuite les éléments et finalement la matière, substance 
modelante. L'assemblage particulier ainsi que la composition principale ${ }^{3}$ de cette matière permettent de la qualifier d'organique: «la vie est une propriété émergente de l'organisation de la matière, longuement mûrie par la surface de la terre, océans, continents et atmosphère, et les diverses étapes qualitatives des formes d'organisation des êtres vivants peuvent chacune apparaître aussi comme telles. ${ }^{4}$.

Donc, ce que je suis est en grande partie la conséquence heureuse d'un agencement systémique de particules fondamentales. Mon corps est une entité organisée en plusieurs systèmes; d'abord en systèmes physiques, ce qui détermine la structure; ensuite en systèmes biologiques, ce qui détermine la vie, donc le sensible. Car la vie, au contraire de l'inanimé, devient synonyme d'échanges : le corps humain est un système ouvert et dynamique de par les millions d'échanges s'effectuant entre l'origine des cellules le composant et l'environnement dans lequel il baigne : «L'interaction entre les électrons et les noyaux atomiques esí donc à la base de tous les corps solides, liquides et gazeux, ainsi que de tous les organismes vivants et des processus biologiques qui leurs sont associés.m. ${ }^{5}$.

En projetant cette analogie au théâtre, j'ai toujours imaginé le performer, dans son univers scénique, comme un système atomique: l'atome-performer. Son corps est

\footnotetext{
${ }^{3}$ La matière organique est principalement composée de carbone, d'hydrogène, d'oxygène, d'azote et de phosphore avec des traces minérales comme le fer et le calcium. (C,H,O,N,P, Fe, Ca)

${ }^{4}$ Pary, M. (2003). La physique du XXe siècle. Collection Sciences \& histoires. France: EDP Sciences, page 240

${ }^{5}$ Capra, F. (1975). Le tao de la physique. Paris : Éditions Sand, page 74.
} 
un noyau très dense d'énergie, qui, s'alliant aux multiples formes d'énergie environnantes (sonore, lumineuse, visuelle), excite l'autre système atomique présent: l'atome-spectateur. Dans certaines conditions, ces deux atomes échangent et parfois « fusionnent » lors de l'évènement scénique; ils vibrent en symbiose et forment une sorte de molécule théâtrale. Cette molécule est envisagée comme un système, définitivement vivant, donc sensible et ouvert, en échange constant entre les divers stimuli environnants et ceux qui la composent. Cette molécule représente la matière fondamentale de l'évènement scénique, la substance matricielle de l'expérience sensible.

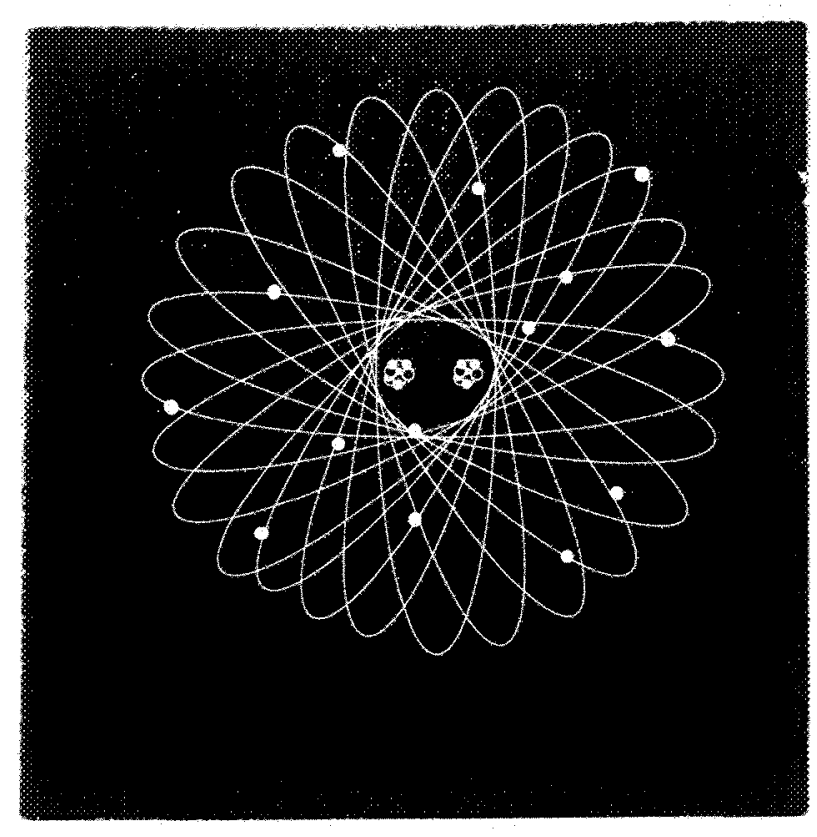

Figure 3. Molécule théâtrale

Cette comparaison entre l'art et la science traduit tout à fait mes préoccupations systémiques. Elle sert aussi, bien entendu, mon propos physiologique; elle démontre de 
toute évidence à quel point cette manière de concevoir le monde ne tient pas seulement du simple exercice atomique, mais plutôt d'un réflexe acquis, ancré profondément en moi.

\section{L'approche systémique}

C'est dans cet esprit, lorsque j'ai découvert l'approche systémique dans le cadre de mes cours avec madame Élizabeth Kaine et madame Diane Laurier, que cette méthode s'est imposée comme une révélation pour la conduite de cette recherche d'ordre plus fondamental : «En optant pour une approche systémique, l'objectif de la recherche devient de représenter ou de modéliser la complexité que l'on cherche à saisir ou encore à interpréter. $»^{6}$. Pour cette raison, puisqu'elle devient un outil de modélisation, il m'apparât important de la définir dans ce chapitre.

Selon Joël de Rosnay, cette méthodologie n'est ni une science, ni une théorie, ni même une discipline; elle est qualifiée d'approche "permettant de rassembler et d'organiser les connaissances en vue d'une plus grande efficacité sur l'action. $»^{7}$. Elle se définit comme une "méthode d'analyse et de synthèse prenant en considération

\footnotetext{
Laurier, D. Notes de cours sur la méthodologie de la recherche. (Automne 2004) UQAC.

${ }^{7}$ De Rosnay, J. (1975). Le macroscope : vers une vision globale. Paris : Editions du Seuil . page 91
} 
l'appartenance à un ensemble et l'inierdépendance d'un système avec les autres systèmes de cet ensemble $»^{8}$.

Rosnay explique que l'approche systémique est apparue dans les cinquante dernières années, issue du métissage de plusieurs disciplines telles que la biologie, la théorie de l'information, la cybernétique et la théorie des systèmes. Dans son livre intitulé Le Macroscope, il spécifie qu'« à la différence de l'approche analytique, l'approche systémique englobe la totalité des éléments du système étudié, ainsi que leurs interactions et leurs interdépendances.»".

Ces deux approches, analytique et systémique, sont davantage complémentaires qu'opposées. L'approche analytique cherche à réduire un système à ses éléments constitutifs les plus simples afin d'étudier le type d'interaction entre eux, ainsi que leurs détails. L'approche systémique souhaite plutôt envisager le système dans sa totalité, dans sa complexité et dans la dynamique qui lui est propre. Cette dernière option permet de dégager les propriétés d'un système, qui ne sont jamais applicables aux éléments individuels le composant. Ce qui, je crois, est tout à fait le cas de la molécule théâtrale.

\footnotetext{
${ }^{8}$ Tel que défini par De Villiers, M. Multidictionnaire de la langue française ( $4^{\mathrm{e}}$ édition) Québec : Éditions Québec Amérique.

${ }^{9}$ De Rosnay, J. (1975). Le macroscope : vers une vision globale. Paris : Éditions du Seuil., page 92.
} 
D'ailleurs, un clivage entre le monde cartésien et sa nature très analytique s'est effectué en grande partie lors du passage de notre société au paradigme systémique, selon Jean-Louis Le Moigne ${ }^{10}$.

Rosnay dégage trois principes de l'approche systémique qui justifient, par le faitmême, son utilité. Premièrement, s'élever pour mieux voir; avoir une vision globale de ce que l'on étudie. Deuxièmement, relier pour mieux comprendre; faire des liens, découvrir les interactions entre les différents éléments d'un système. Finalement, situer pour mieux agir; mettre les objets dans les bonnes boites et organiser les connaissances, ce qui implique nécessairement une plus grande efficacité de l'action. Voilà, en général, les buts de l'approche systémique.

\section{L'objet « système»}

La notion de «système » se trouve au cœur de l'approche systémique. Rosnay spécifie d'ailleurs qu'aucune définition ne peut être satisfaisante, que seule la notion de système est féconde. $\grave{A}$ cela, il ajoute que le concept de système est un «ensemble d'éléments en interaction, organisés en fonction d'un but. " " . Pour moi, l'évènement scénique et ses constituants correspondent à cette notion.

\footnotetext{
${ }^{10}$ Le Moigne, J-L. (1977). La théorie du système général: théorie de la modélisation. Paris: Presses universitaires de France. Page 30

${ }^{11}$ De Rosnay, J. (1975). Le macroscope : vers une vision globale. Paris : Editions du Seuil Page 100
} 
Le Moigne ajoute une triangulation à cette notion de système: Structure Activité - Évolution. Voici d'ailleurs la figure qui représente l'objet « système» dans le paradigme systémique. C'est «un objet qui, dans un environnement, dotés de finalités, exerce une activité et voit sa siructure interne évoluer au fil du temps, sans qu'il perde pour autant son identité. $\rangle^{12}$.

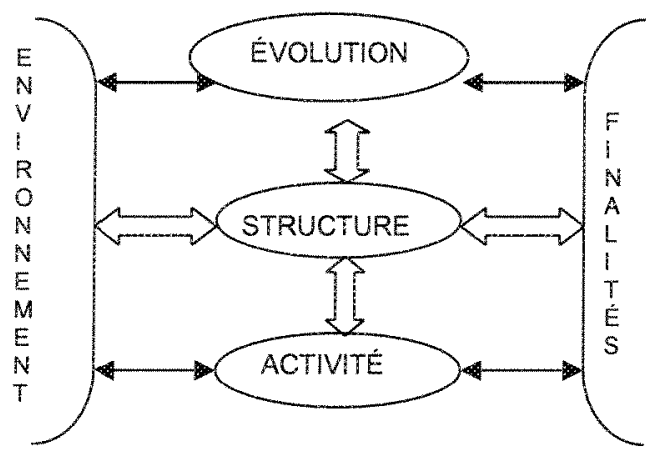

Figure 4. Modélisation du paradigme systémique selon Jean-Louis Le Moigne

Tout système peut être décomposé en deux aspects fondamentaux: l'aspect structural, c'est-à-dire l'organisation du système, de ses composants et/ou éléments dans l'espace, son organisation spatiale propre; et l'aspect fonctionnel, c'est-à-dire l'organisation temporelle du système, tout les processus et phénomènes qui sont liés, dépendants du temps. Les principaux exemples de l'aspect fonctionnel sont les échanges, les transferts, le flux, la croissance, l'évolution.

\footnotetext{
12 Le Moigne, J-L. (1977). La théorie du système général : théorie de la modélisation. Paris : Presses universitaires de France, page 36.
} 
Plusieurs propriétés, intemes et/ou extemes s'appliquent également aux systèmes en général. L'ouverture et la complexité, qui sont deux propriétés fondamentales, m'intéressent particulièrement pour les systèmes traités dans cette recherche.

En effet, un système ouvert est nécessairement de très haute complexité : le système ouvert (SO) «échange énergie, matière et informations utilisées dans le maintien de son organisation contre la dégradation qu'exerce le temps $\rangle^{13}$. II est en relation permanente avec son environnement, son écosystème, l'un modifiant l'autre et l'autre se trouvant modifié en retour.

Bemard Walliser précise: «L'ouverture du système a essentiellement pour conséquence que son comportement dépend de moins en moins de sa propre constitution, mais de l'environnement dans lequel il baigne, le système ne pouvant plus être, à la limite, qu'un reflet de son environnement. $"{ }^{14}$.
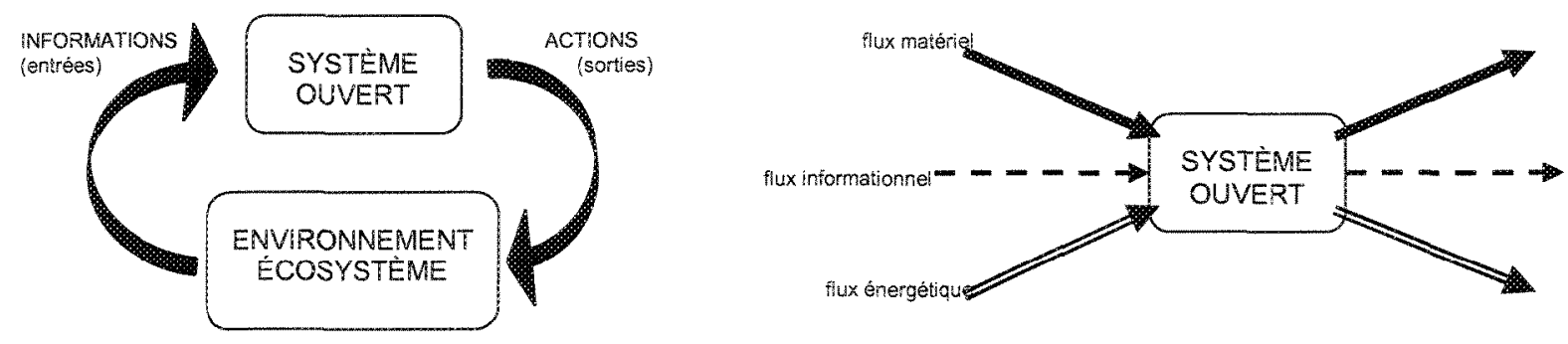

Figure 5. Ouverture d'un système

\footnotetext{
${ }_{13}^{13}$ De Rosnay, J. (1975). Le macroscope : vers une vision globale. Paris : Éditions du Seuil, page 102.

14 Walliser, B. (1977). Systèmes et modèles: introduction critique à l'analyse de systèmes. Paris: Éditions du Seuil, page 33.
} 
La complexité est définie, dans Le Macroscope, selon quatre axes principaux. Un système ouvert possède une grande variété d'éléments avec des fonctions spécialisées : il dénombre plusieurs niveaux hiérarchiques internes; il est caractérisé par une haute densité d'interconnexions, due à la grande variété de liaisons entre les différents niveaux et éléments; en dernier lieu, les interactions d'un système ouvert complexe sont nonlinéaires.

Chaque système peut s'organiser à différents niveaux hiérarchiques, en diverses couches, selon les relations entre les sous-systèmes le composant et les autres systèmes liés à lui. Il apparaît alors un ordre d'emboîtement traduisant des échelles différentes d'appréhension des phénomènes que l'on cherche à modéliser.

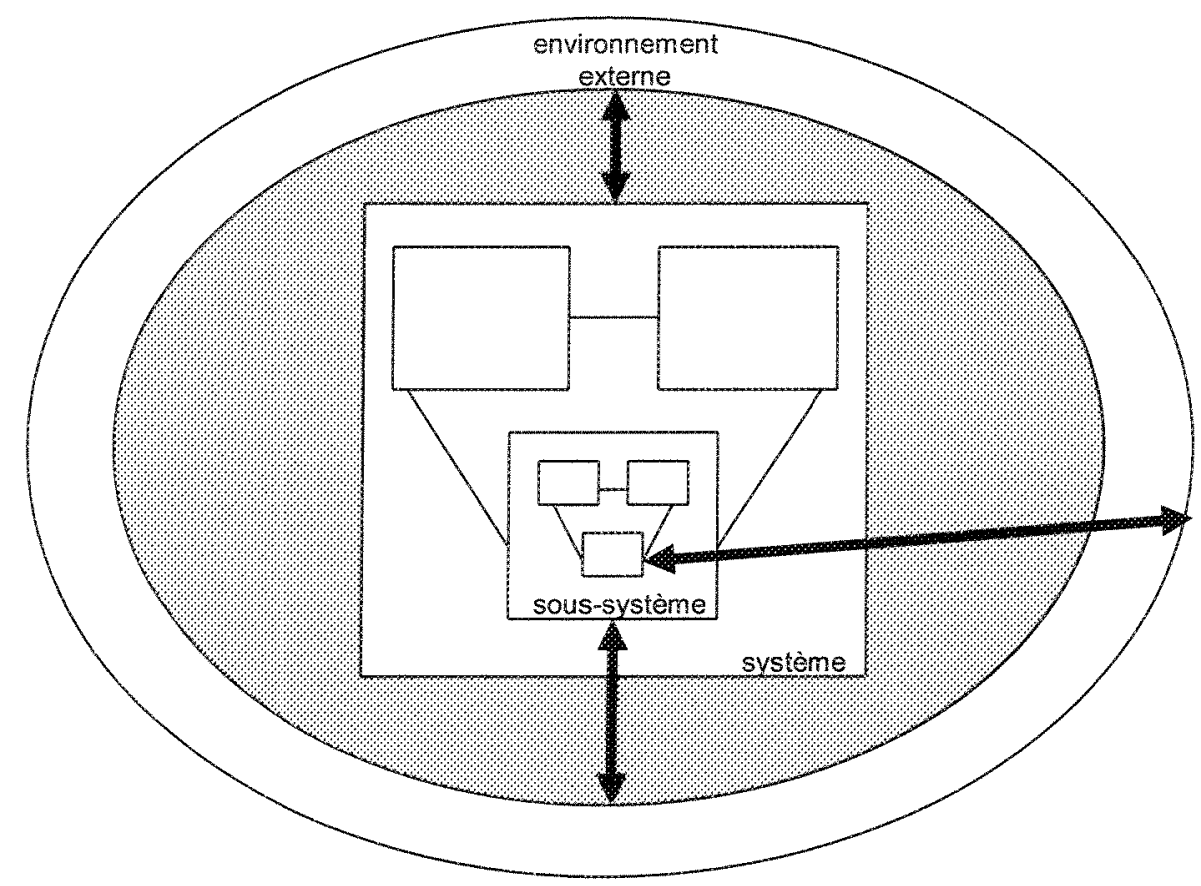

Figure 6. Exemple de relations, de niveaux hiérarchiques entre système et sous-systèmes selon Bernard Walliser 
L'approche systémique définit done la méthodologie suivant laquelle cette recherche va se développer: elle tentera de modéliser l'évènement scénique et l'expérience sensible du spectateur au niveau physiologique. Elle cherchera à confronter les concepts, les structures se situant à différents niveau d'abstraction (artistique, physiologique) afin de créer une représentation dynamique du système théâtral. Une représentation de la vie, de cet art de relation, de cet art vivant exceptionnel qu'est le théâtre. 
CHAPITRE II

L'ethnoscénologie comme macrosystème théorique de l'évènement scénique 
Ce chapitre représente le cadre théorique de la recherche et cherche à la contextualiser par le recours à l'ethnoscénologie. Suivront les notions-clé utilisées pour la définition de l'évènement scénique. Enfin cette partie sera conclue avec une référence au théâtre postdramatique, puisque cette recherche s'inscrit à l'intérieur de ce courant.

\section{Ethnoscénologie}

Les racines de mon intérêt pour le théâtre organique se trouvent dans l'ethnoscénologie, la « science » qui étudie les spectacles vivants.

Cette discipline, relativement nouvelle, est abordée ici parce qu'elle semble s'écarter d'un certain intellectualisme théorique en décrivant le spectacle vivant comme un évènement biologique exceptionnel, ce qui correspond parfaitement à mon questionnement de départ: que se passe-t-il dans le corps du spectateur?

Il existe très peu de théories portant sur le théâtre physiologique, sur cet échange, cette mise en présence d'organismes vivants dans ce contexte particulier. L'ethnoscénologie s'est donc présentée comme un cadre guidant et circonscrivant l'exploration d'un théâtre physiologique. 
Cette théorie, proposée entre autres par Jean-Marie Pradier, se consacre à l'étude des relations entre l'art ế la science, et plus précisément entre les pratiques et comportements humains spectaculaires organisés (PCHSO) - dont le théâtre et la danse sont des sous-ensembles culturels -, les sciences de la vie et les sciences de la matière. Pradier spécifie: «par spectaculaire, il faut comprendre une façon d'être, de se comporter, de se mouvoir, d'agir dans l'espace, de s'émouvoir, de parler, de chanter et de s'orner qui tranche sur les activités banales du quotidien ou les enrichit et fait sens. (...) Le mot spectaculaire (performing en anglais) dans PCHSO : 1) ne se réduit pas au visuel; 2) se réfêre à l'ensemble des modalités perceptives humaines; 3) souligne l'aspect global des manifestations expressives humaines, incluant les dimensions somatiques, physiques, cognitives, émotionnelles et spirituelles.» ${ }^{15}$.

L'ethnoscénologie comprend l'analyse des modalités selon lesquelles les pratiques et les comportements humains spectaculaires organisés s'insèrent dans leur cadre socioculturel. Elle comprend également l'approche des stratégies cognitives qui sous-tendent l'émergence de ces comportements et pratiques. Enfin, elle se consacre à l'étude des éléments qui constituent les modèles systémiques des pratiques et des comportements humains spectaculaires organisés. C'est particulièrement ce troisième volet qui nous intéresse.

\footnotetext{
${ }^{15}$ Pradier, Y.-M. (1995). Ethnoscénologie, manifeste, Théâtre/Public, \#123, pages 46-48.
} 
Les champs d'investigation de l'ethnoscénologie englobent donc l'incroyable diversité des pratiques et des comportements humains spectaculaires organisés. Cependant, avec une approche systémique, elle place le corps au centre de ses préoccupations. Elle «suggère la dimension organique de l'activité symbolique et sounaite montrer l'extrêne vitalité, la complexité et l'interactivité des dimensions constitutives de l'être humain. ${ }^{16}$. Une partie de l'ethnoscénologie tente de cerner ce qui se passe lors de l'échange entre deux corps; son matériau principal est le réel physiologique. L'intérêt pour les arts vivants que sont par exemple le théâtre, la danse et le cirque, constitue l'identité spécifique de l'ethnoscénologie. Quoi de mieux alors pour aider à saisir et à modéliser l'expérience sensible du spectateur?

Jean-Marie Pradier affirme: «Un spectacle vivant constitue un évènement biologique exceptionnel. Le corps s'y retrouve dans son monde au point d'articulation des fondements biologiques universels des comportements et des apprentissages culturels des participants. $"{ }^{17}$. II nous amène donc dans le domaine du signal, au sens physique du terme; les molécules, vibrations, ondes, phéromones et photons qui habitent la mise en présence de deux organismes vivants sont partie prenante de l'expérience spectaculaire. Il nomme ces signaux «biosèmes »" Ces notions sont à l'opposé du signe et de la sémiotique, qui jusqu'ici demeurent les principales portes d'entrée de la théorie sur la réception théâtrale, où «la scène est perçue comme un système de signes et

\footnotetext{
${ }^{16}$ Pradier, J.-M. (1994). Le public et son corps : Éloge des sens. Théâtre/Public, \#120, pages 18-33.

${ }^{17} \mathrm{Ibid}$.

${ }^{18}$ Ibid.
} 
non comme un système de corps. $)^{19}$. En choisissant de s'intéresser aux stimuli et aux signaux de l'évènement, cette recherche jette donc un regard sur la scène avant tout comme un «système de corps».

Le système phanique de Jean-Marie Pradier est un exemple fort lumineux de la scène comme un système de corps:

Il permet de réévaluer la compréhension du spectaculaire et son importance pour les organismes vivants. (...) I'ai appelé système phanique la dynamique de l'interaction qui, au niveau biologique, est engendrée par la mise en présence réelle de deux organismes vivants. Système éminemment complexe dont nous sommes loin, encore aujourd'hui, d'avoir achevé l'inventaire: l'arbre des causalités cultivé par les neurosciences ne cesse de se ramifier. Les modifications toni-posturales, neuro-hormonales induites par la mise en présence de deux êtres vivants aussi achevés que l'homme ont leur racines dans les filiations animales et la mémoire de l'espèce. Les apprentissages culturels - dont le langage - maquillent assurément l'archaïsme des réponses phaniques en opérant une sélection explicite partielle de ce qui fait sens. ${ }^{20}$

\footnotetext{
${ }^{19}$ Pradier, J.-M. (1994). La scène des sens ou les voluptés du vivant. Internationale de l'imaginaire, \#2, pages 13-32.

${ }_{20}$ Pradier, J.-M. (1994). Le public et son corps : Éloge des sens. Théâtre/Public, \#120. pages 18-33.
} 
L'ethnoscénologie ${ }^{21}$ pose donc des balises et justifie mon envie de modéliser l'expérience sensible du spectateur, plus précisément dans le contexte d'un évènement scénique d'art performatif. Elle constitue, en quelque sorte, le macrosystème dans lequel s'intègreront les systèmes relatifs à l'évènement scénique.

Il est maintenant nécessaire de tracer les limites de notre propos de recherche à l'intérieur de ces champs théoriques et de mieux définir l'évènement scénique avec tous les microsystèmes qui circonscrivent l'expérience sensible du spectateur.

\section{L'évènement scénique}

Dans ce laboratoire qu'est l'évènement scénique, deux organismes vivants sont mis en présence. Ces deux corps communiquent, échangent, réagissent l'un à l'autre d'une façon consciente et inconsciente. Plus précisément: qu'est-ce que le corps du spectateur reçoit, comment gère-t-il ce flux d'énergie, d'informations de toutes sortes,

\footnotetext{
21 À des fins de précision, voici un extrait du manifeste de l'ethnoscénologie, qui permetra de clore le portrait des origines de cette discipline. «Le Centre International d'Ethnoscénologie, placé sous les auspices de l'UNESCO, a été créé en 1995, à l'initiative de deux institutions: la Maison des Cultures et du Monde qui est un lieu permanent d'échanges et de dialogue entre les formes d'expression et les identités culturelles des peuples du monde. Cette Maison est présidée par le professeur Jean Duvignaud et dirigée par Cherif Khaznadar et Françoise Gründ; elle publie la revue L'Internationale de l'Imaginaire. L'autre institution fondatrice est l'équipe de recherche du Laboratoire interdisciplinaire des pratiques spectaculaires de l'Université Paris 8. Cette équipe est dirigée par le professeur Jean-Marie Pradier, membre permanent de l'International School of Theater Antropology (dir. Eugenio Barba). Les activités du Centre International d'Ethnoscénologie s'organisent autour de trois rubriques: la recherche, l'enseignement et la diffusion».

Tiré de : Pradier, J-M. Ethnoscénologie, manifeste. Theâtre/Public, \#123, pages 46-48.
} 
provenant à la fois du performer devant lui et, simultanément, de tout le système scénique en général?

Peu importe la façon dont l'espace théâtral, le lieu physique, est disposé, le corps du spectateur est au centre d'une impressionnante tempête sensorielle: «(il) est nommément reconnu partenaire dans l'évènement théâtral, non en tant qu'entité distincte mais comme élément indissociable d'un système vivant hypercomplexe. $»^{22} .11$ en fait partie parce qu'il reçoit des stimuli, mais également parce qu'il en produit. Bien sûr, cette expérience sensible ${ }^{23}$ est influencée par une foule de facteurs, certains déterminants, d'autres plus secondaires, et se vit dans une ouverture totale à la société et au monde. Cependant, pour cette recherche, il faut tracer certaines frontières, qui demeureront toutefois très ouvertes et perméables, afin de pouvoir modéliser l'expérience sensible et être en mesure de montrer qu'il se produit bien un phénomène d'empathie biologique chez le spectateur lors de l'évènement scénique.

Un moment vivant, une mise en présence simultanée de deux organismes dans un lieu et un temps donné, avec toutes ses conséquences, voilà une définition assez vaste et personnelle de l'évènement scénique. Afin de la resserrer, nous ajouterions «dans un contexte scénique». "Scénique» désigne ici les pratiques spectaculaires telles que définies par l'ethnoscénologie et, de façon plus précise encore, le théâtre, la danse, l'art performatif, c'est-à-dire toutes les formes d'utilisation du corps en direct, dont la

\footnotetext{
${ }^{22}$ Pradier, J.-M. (1994). Le public et son corps: Éloge des sens. Théâtre/Public, \#120, pages 18-33.
}

${ }^{23}$ Le mot sensible est utilisé au sens physiologique; sensation perçue et traitée par le système nerveux. 
majeure partie se produit suivant une volonté organique, de façon à constituer une réelle stimulation pour le corps du spectateur.

Le rapport scène-salle traditionnel, à l'italienne, n'est pas l'unique et nécessaire cadre de cet évènement dans l'espace. Tout autre rapport entre le performer et le spectateur dessine une nouvelle perception dans le corps de ce dernier. Pour moi, l'évènement scénique circonscrit les multiples formes d'échanges possibles, de façon consciente ou non, entre deux corps, celui du performer et celui du spectateur. Elle englobe la totalité des stimuli qui parviennent au spectateur, visuels, auditifs, tactiles et kinesthésiques lorsqu'il assiste à de l'art performatif.

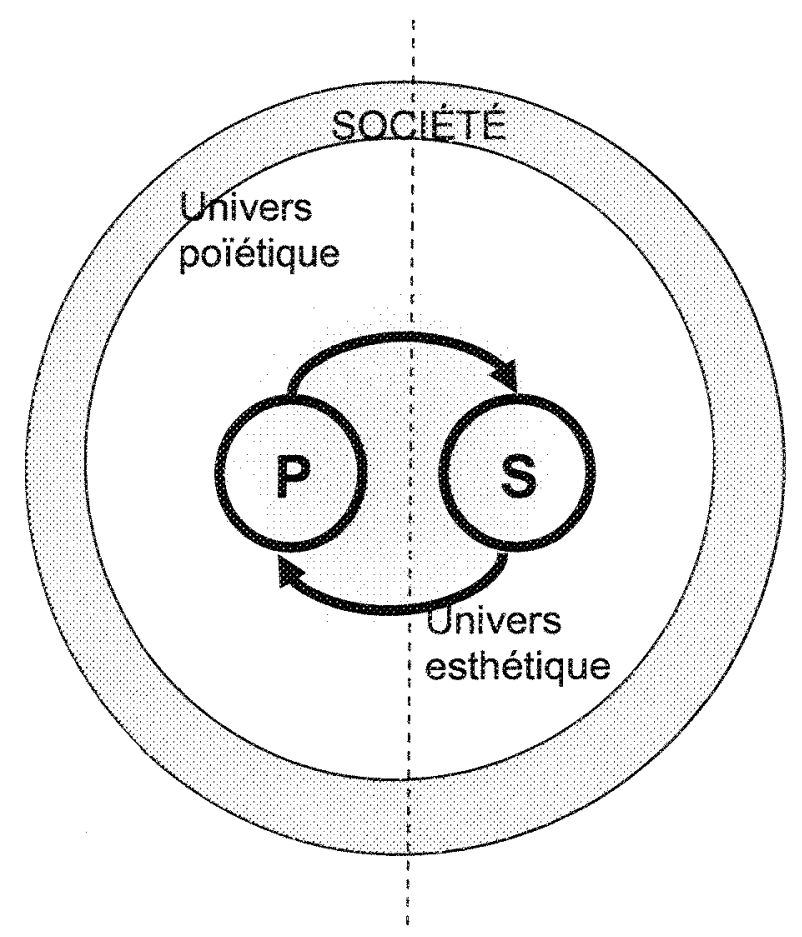

Figure 7. Modèle de l'évènement scénique 


\section{Le performer et l'organicité}

Une première limite s'impose ici. L'importance de l'organicité est, selon nous, capitale pour vivre une expérience sensible. En effet, toute communication, toute forme d'échange physiologique est quasi réduite à néant s'il n'y a pas d'implication organique, de conscience corporelle des vivants mis en présence, autant de la part du spectateur que chez le performer.

L'organicité est une «activité autonome, spontanée, authentique, un flux de la vie qui s'écoule dans le corps. Pour devenir un réel acte performatif, elle doit néanmoins être saisie par une conscience qui restitue l'invisible dans le visible, et le spontané dans le structuré.. ${ }^{24}$.

Pour cette raison, il convient d'établir, selon Maria Leão et Jean-Marie Pradier, une différence entre l'art spectaculaire, qui est produit en fonction du résultat (représentation dirigée vers les spectateurs, la perception attendue), et l'art performatif, où l'accent est mis sur le processus intérieur, organique, de celui qui fait, de l'artiste. Le performer n'est pas dans la production de signes compréhensibles pour le public, mais bien dans le faire, dans l'organicité, ce qui laisse beaucoup plus de place à la résonance dans le corps du spectateur.

${ }^{24}$ Leão, M. (2003). La présence totale au mouvement. France : Editions Point d"Appui, page 54. 


\section{L'art performatif et l'esthétique de la stimulation}

Pradier distingue également l'esthétique de la simulation fondée sur la narration et une esthétique de la stimulation, qui présuppose l'activation de l'organisme du spectateur pris dans une action qu'il perçoit comme réelle. "Lorsque la quête d'un performer touche un processus réel, impliquant toutes les ressources de son être rassemblées dans un acte total, le speciateur, en amont de la compréhension, résonne dans une empathie organique avec les processus physiologiques éprouvés par le performer. $\gg^{25}$.

Il ne s'agit pas d'offrir au spectateur une image, un simulacre de la vie quotidienne, mais plutôt, d'une façon généreuse et risquée, une présence palpable, un être vivant, un corps matériel, un organisme mouvant, un signal physique, une action : «cette action réelle dont parle Barba est un évènement qui a lieu lors d'une rencontre non-médiatisée ni différée entre des personnes, selon un protocole qui permet aux uns de déployer leur corps, leurs voix, leur souffle et aux autres de les absorber (...). $»^{26}$.

Une autre limite de cette recherche s'affirme ici. Je crois que l'expérience sensible a plus de chance de se produire dans ce contexte d'art performatif, d'esthétique de la stimulation, d'une quête réelle où l'organicité, le corps total de l'acteur est engagé,

${ }^{25}$ Leão, M. (2003). La présence totale au mouvement. France : Éditions Point d'Appui, page 47.

${ }^{26}$ Pradier, J.M. (1994). Le public et son corps : Éloge des sens. Théatre/Public, \#I20, pages 18-33. 
produisant une charge communicative plus forte corporellement. "Restaurer l'organicité du spectacle vivant revient à entrevoir une communication qui se trouve en amont de la dualité signifiant/signifié, (...) qui ne se restreint pas au traitement d'images visuelles mais qui englobent l'ensemble des modalités perceptives, ouvrant une voie de résonnance organique qui touche au plus profond de la mémoire et de l'imaginaire. ${ }^{27}$.

\section{La subjectivité dz spectateur}

L'expérience sensible est grandement influencée par la subjectivité du spectateur. Il est clair qu'aucun spectateur n'aura la même expérience sensible; ni par rapport aux autres vivants présents, ni en référence à ses propres expériences passées lors d'un spectacle. D'innombrables facteurs peuvent teinter cette forme d'expérience physiologique très subjective : la mémoire affective, l'état du moment, l'ouverture et la disponibilité, la fatigue, les souvenirs, les connaissances, l'endroit où l'on se trouve, etc. Cette liste pourrait se poursuivre encore. Le spectateur idéal n'existe donc pas, ni par rapport à l'évènement scénique auquel il assiste, ni par rapport à ce que le metteur en scène et/ou le performer souhaite transmettre.

Par contre, il faut qu'il soit disposé, prêt à s'abandonner. Il faut qu'il prenne autant de risques, d'une certaine manière, que le performer devant lui. Le spectateur doit

\footnotetext{
${ }^{27}$ Leão, M. (2003). La présence totale au mowvement. France : Éditions Point d'Appui, page 41.
} 
faire sa part du travail pour qu'il y ait une véritable communion organique et se muter en système ouvert lors de l'évènement scénique :

(...) nous sommes progressivement passés avec les arts du spectacle vivant de l'wil à la peau, du sens de la vue au tactile (...) nous avons négligé la dimension du contact dont l'expérience ou l'éprewve nous éveille à ce que Merleau-Ponty appelait la "chair du monde». Le théatre, lieu où l'on voit? Plutôt: lieu où l'on touche, lieu où l'on hume. ${ }^{28}$

\section{Théâtre postdramatique}

En cherchant à contextualiser cette recherche, j'ai été forcée d'admettre qu'elle s'inscrivait parfaitement dans ce qu'on appelle aujourd'hui le théatre postdramatique. C'est pourquoi une brève introduction à cette notion me semble importante, puisqu'elle justifie également la valeur de mon questionnement, son ancrage dans cette société d'aujourd'hui.

La définition que donne Hans-Thies Lehmann du théâtre postdramatique place résolument l'être humain, le corps, au centre du dispositif. Cette matière vivante, organique, ne se retrouve plus seulement sur la scène, mais aussi dans la salle, dans l'entité que forme le spectateur. Lehmann reconsidère la place du spectateur, son importance, sa présence participative dans l'espace commun partagé au thêâtre.

\footnotetext{
${ }^{28}$ Pradier, J.-M. (1994). Le public et son corps: Éloge des sens. Théâtre/Public, \#120, pages 18-33.
} 
Le postdramatique n'est ni un genre, ni une forme, ni même un modèle; le concept doit plutôt être envisagé comme une "galaxie", "comme un spectre, large avec des formes diverses ${ }^{29}$. Ce spectre théâtral est axé résolument sur le présent, même sur la présence dans ce qu'elle a de sensible. 11 « délaisse la mimétique et l'illusion du drame pour réinvestir sa spécificité d'acte et d'expérience $»^{30}$, ce qui le rapproche inévitablement de la performance ou du happening, sans toutefois s'y inscrire.

Toujours selon Lehmann, le postdramatique « fait appel à tous les arts : danse, chant, musique, pantomime, théâtre parlé, art graphiques, lumières, vidéo, images virtuelles, hologrammes... $»^{31}$. C'est ce genre de multidisciplinarité que je revendique ici, avec l'évènement scénique. Tous ces stimuli contribuent à la réception physiologique chez le spectateur. Il affirme également que «le pas vers le théatre postdramatique est franchi lorsque tous les moyens théâtraux au-delà du langage se trouvent mis sur un pied d'égalité avec le texte, ou peuvent être systématiquement pensés sans lui. $\rangle^{32}$. Ces moyens donc, y compris le texte, constituent, pour cette recherche des stimuli, des signaux parvenant au corps du spectateur.

Lehmann précise enfin que le thêâtre postdramatique se veut «davantage présence que représentation, davantage expérience partagée qu'expérience transmise,

29 Biet, C., \& Triau, C. (2006). Qu'est-ce que le théatre? Collection Folio Essais. France : Éditions Gallimard.

${ }^{30}$ Lehmann, H.-T. (2002). Le théâtre postdramatique. Paris : Éditions de l'Arche.

${ }^{31}$ Ibid

${ }^{32} \mathrm{Ibid}$ 
davantage processus que résultat, davantage manifestation que signification, davantage impulsion d'énergie qu'information. $\rangle^{33}$. Voilà en quoi je me sens ancrée dans ce courant.

En résumé, l'évènement scénique, dans le cadre de l'ethnoscénologie et du théâtre postdramatique, est un échange sous forme d'art performatif, engagé dans une organicité et une esthétique de la stimulation entre le performer et le spectateur. $\mathrm{Ce}$ demier se doit d'être un système ouvert afin de favoriser l'occurrence de l'expérience sensible.

\section{Une pratique performative organique et systémique : le buto}

Ce cadre théorique se terminera avec une illustration. Ayant eu la chance de vivre quelques ateliers de buto, cette pratique est, à mon sens, une parfaite concordance des notions élaborées précédemment et une source d'inspiration personnelle.

Le buto est un exemple incontestable d'art performatif, mais surtout de pratique systémique. Cette danse d'origine japonaise se traduit par la symbiose du performer et de son environnement. Le mouvement résultant provient la résonnance de son corps aux stimuli qui le percutent, de façon interne ou externe. L'échange devient cyclique, impliquant une énorme sensibilité physiologique, une acuité de sa propre corporéité. Ce

\footnotetext{
${ }^{33}$ Lehmann dans : Biet, C., \& Triau, C. (2006). Qu'est-ce que le théâtre? Collection Folio Essais. France : Éditions Gallimard, page 889.
} 
n'est pas un évènement accompli en fonction d'un résultat esthétique, mais plutôt une réelle stimulation de lorganicité du danseur dans son rapport au monde, en tant qu'entité composant et influençant ce même monde. Et tout le corps, dans sa totalité, est mis à contribution : les os, les muscles, les organes, la peau. L'humain qui fait du buto devient un capteur, un restitueur de vie. Sa mémoire physiologique, affective, sa sensibilité, sont exacerbés à un point tel qu’il peut percevoir les molécules d'air se percutant sur sa peau et ce que cela peut produire dans le materiau organique qu'est devenu son corps. En fait, le danseur s'entraine en continu afin d'atteindre cette conscience d'être une partie prenante de l'environnement dans lequel il évolue, respire. Il est un organisme sensible, un système, une totalité ouverte sur le monde, un maillon constituant de l'univers. Par le fait-même d'être en résonnance avec ses extrants et ses intrants, le danseur de buto est, sans aucun doute, l'amorce d'une résonance physiologique chez le spectateur. 


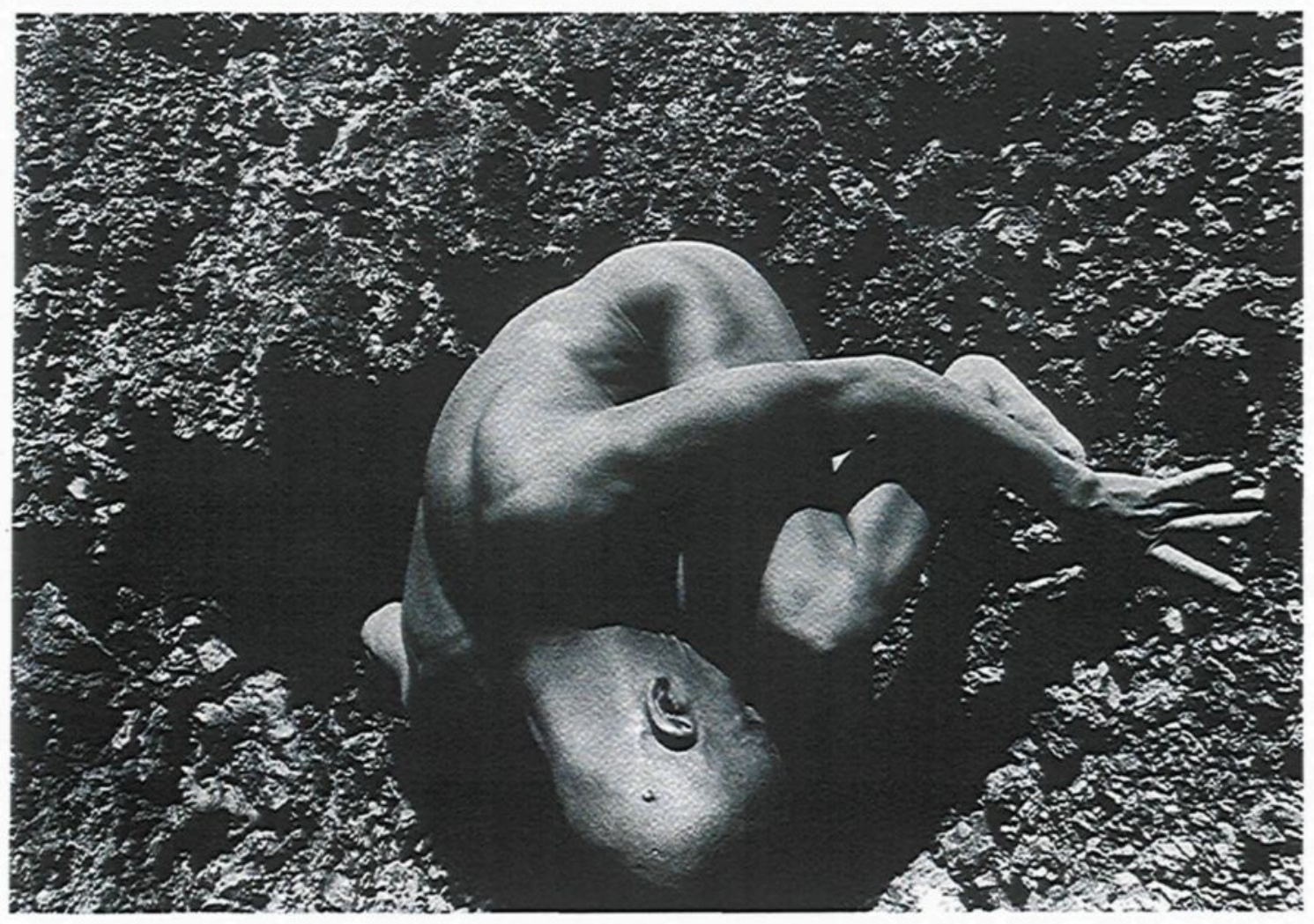

Figure 8. Un exemple incontestable d'art performatif et de pratique systémique. Tanaka Min en Islande, 1980. (photo : Tahara Keiichi). Les photos des figures $8,9,10$ et 11 sont tirées du livre d'Aslan, O. : Butô(s). 


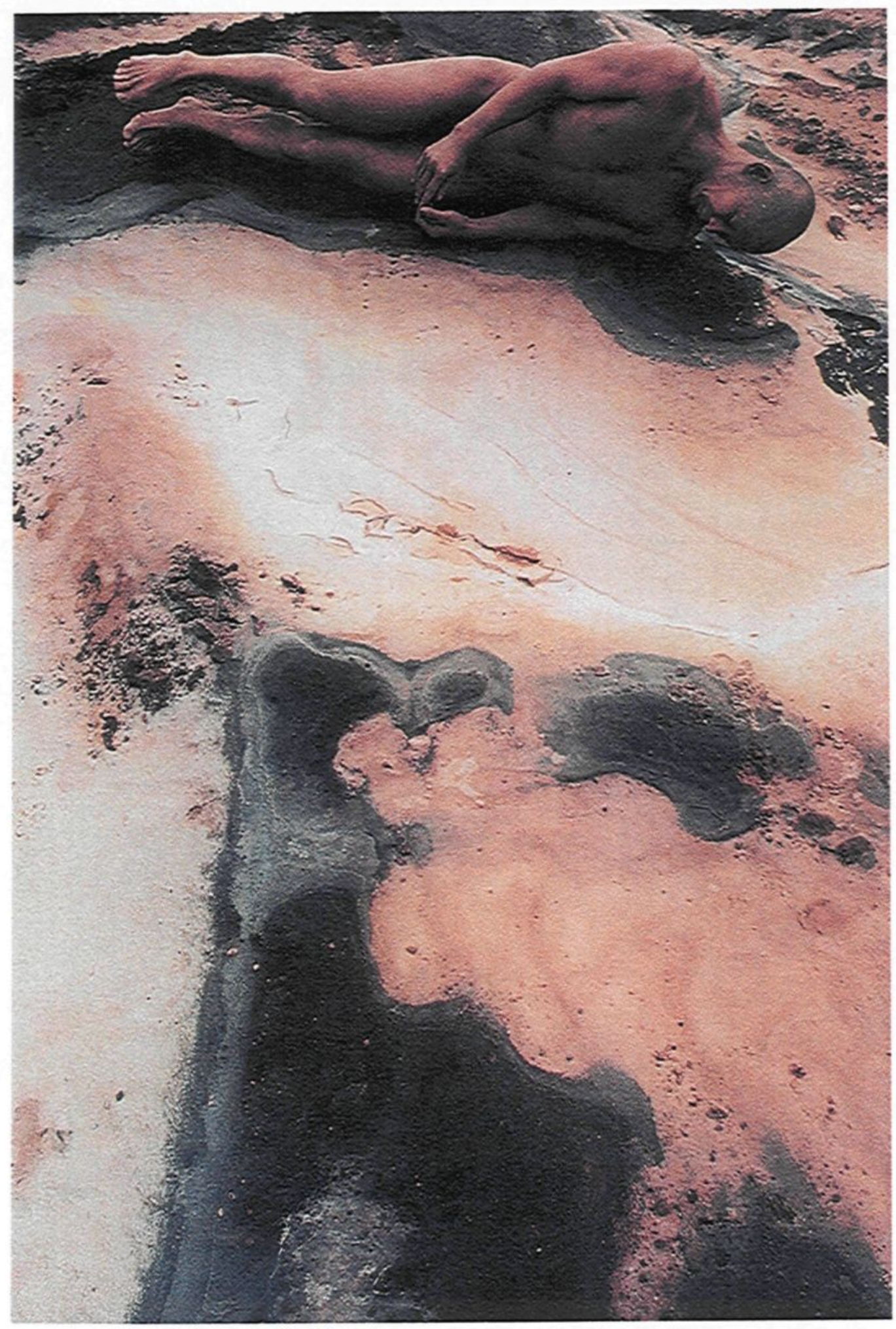

Figure 9. Tanaka Min en Islande, 1980. (photo : Tahara Keiichi) 


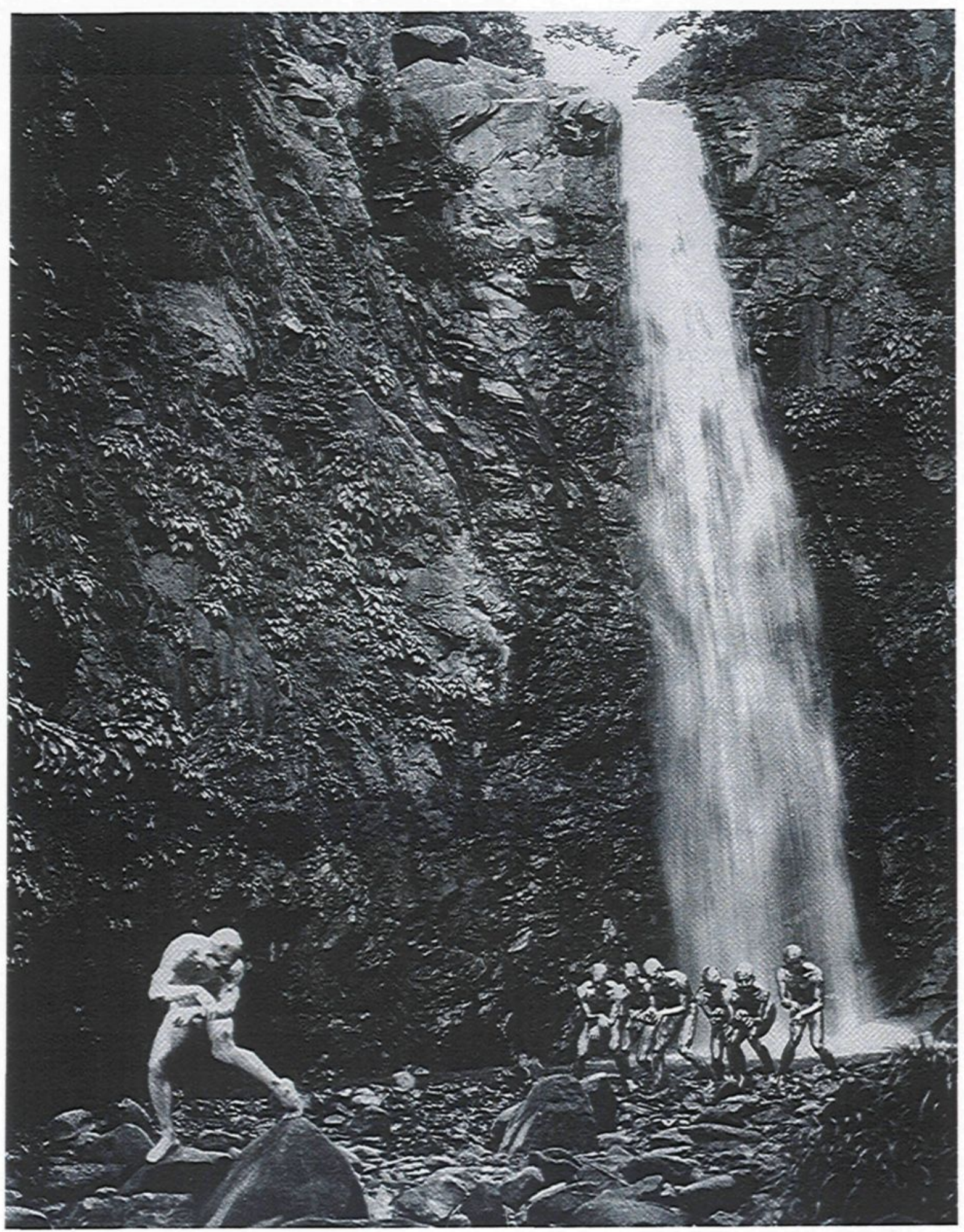

Figure 10. Training dirigé par Murobushi K. au Japon. 


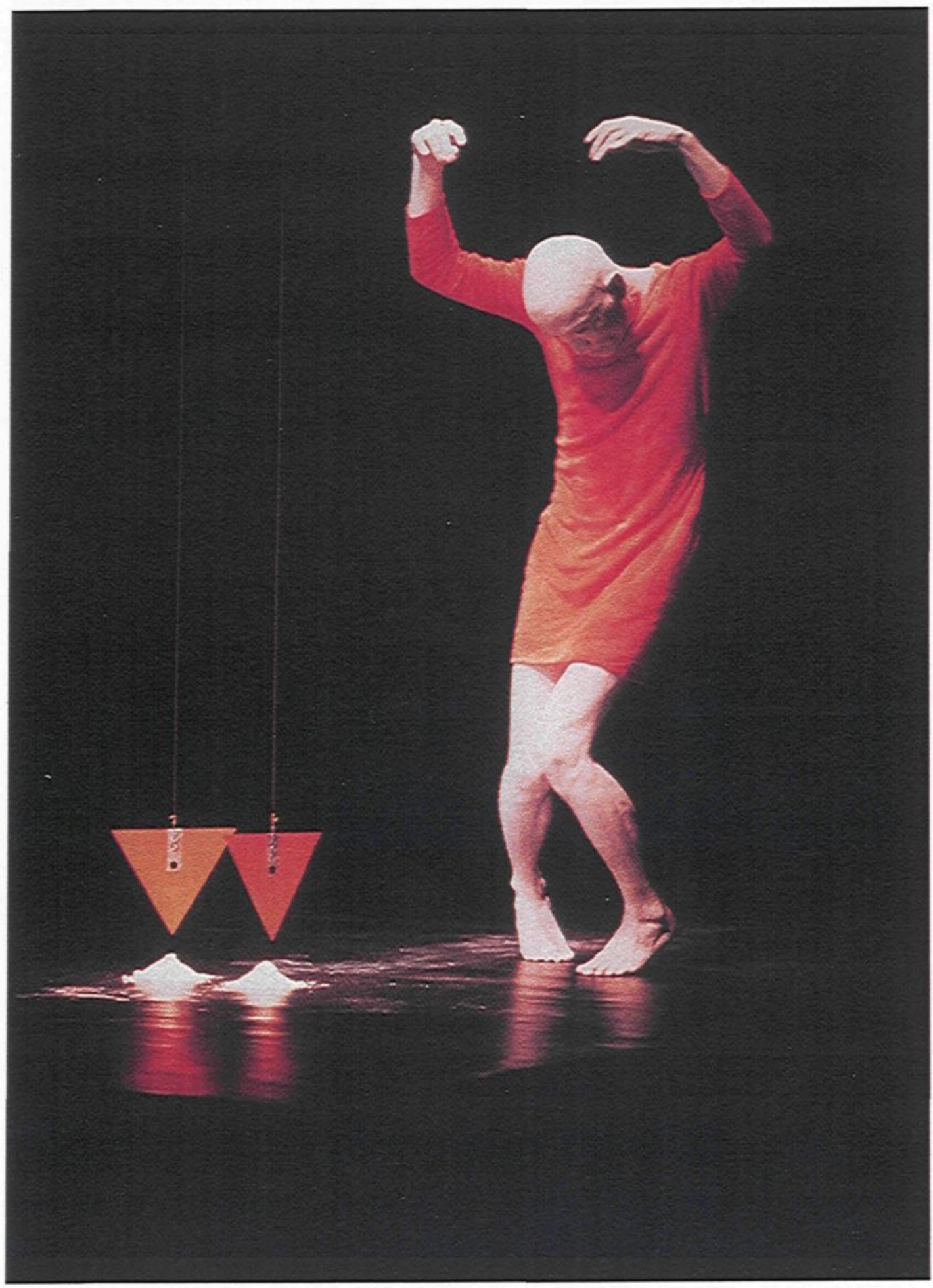

Figure 11. Amagatsu Ushio dans Omote, Théâtre de la ville, Paris, 1991. (photo : Birgit). 


\section{CHAPITRE III}

L'expérience sensible chez le spectateur : de la physiologie à l'empathie 
Disponible, ouverte, je suis là; essayant d'être prête. Je tente de saisir la matière qui se présente à mon corps. Je suis un capteur, une sensibilité exacerbée, aiguisée par tous mes pores. J'absorbe les propriétés physiques pour les muter en organicité; je suis une machine mouvante nourrie au flux communicationnel, à l'énergie. Quel est le chemin atomique qui me fait percevoir? Comment cette matière devient conscience; comment les ondes et les photons deviennent une expérience sensible? Le performer, un noyau irradiant, et moi, la spectatrice, un organisme perméable, en complète osmose avec l'évènement scénique.

L'expérience sensible du spectateur est approfondie ici, dans ses retranchements physiologiques. Cela représente l'aspect le plus fondamental de cette recherche en théâtre. C'est-à-dire que la notion d'interprétation ou même de réception ne sera pas abordée. Cela pourrait représenter le sujet pour bien d'autres recherches encore. L'investigation se limitera à la perception des stimuli par le corps du spectateur: l'inventaire de ces derniers ainsi que la façon dont le corps traite ces stimuli au niveau du système nerveux.

Ce chapitre tentera de répondre principalement à la question: que se passe-t-il dans le corps du spectateur lors d'évènements scéniques d'art performatif? À partir de ce que Pradier a amorcé, les signaux mis en présence lors de l'évènement scénique entre le performer et le spectateur, ou les «biosèmes » comme il les appelle, seront retracés. Comment ces signaux influent-ils et créent-ils l'expérience sensible chez le spectateur? Comment les biosèmes sont-ils intégrés dans le corps de ce dernier? 
Je précise d'emblée à quel point je suis consciente de la complexité de cette expérience qui découle d'un va-et-vient incessant entre le niveau cognitif / affectif et le niveau physiologique. Il est même quasi impossible de la saisir en essayant de soustraire l'un de ces aspects. Cependant, pour les besoins de cette recherche, je me limiterai le plus possible à l'aspect concemant le corps. Je tente d'avoir une vision systémique des systèmes à l'œuvre, une photographie certes éphémère, sachant que l'instant d'après tout peut basculer et que tout se transforme continuellement. Ce point de vue physiologique, qui élimine volontairement certains niveaux de l'expérience sensible, est par contre nécessaire pour entrevoir le reste.

\section{Les signaux ou les biosèmes}

Comme il a été mentionné précédemment, l'évènement scénique est un moment vivant, une mise en présence simultanée de deux organismes dans un lieu et un temps donnés. Un évènement biologique exceptionnel comme nous le rappelle Jean-Marie Pradier. Un évènement riche de sensations, de perceptions et d'émotions, aussi varié et différent pour chaque spectateur qu'il existe d'humains sur cette terre.

Dans le cadre de cet évènement, quels sont les signaux physiques par lesquels circule l'information? Je spécifie encore que ce n'est pas le sens, le signe, qui est recherché, mais bien le signal dans sa matérialité physique, à l'exemple de la scène perçue comme un système de corps. Je laisse la sémiologie à d'autres théoriciens traitant 
de la réception theâtrale, en tentant de viser uniquement l'expérience dans son entité corporelle, organique. Finalement, quels sont ses signaux, à la base de l'expérience sensible du spectateur?

Le signal se définit comme un corps physique ou chimique par lequel se transmet 1'information. Selon ma compréhension subjective de l'évènement scénique, il existe des signaux visuels, auditifs, tactiles et kinesthésiques, olfactifs, même parfois gustatifs. Étant donné la rare présence du goûter au théâtre, cet élément ne sera pas développé.

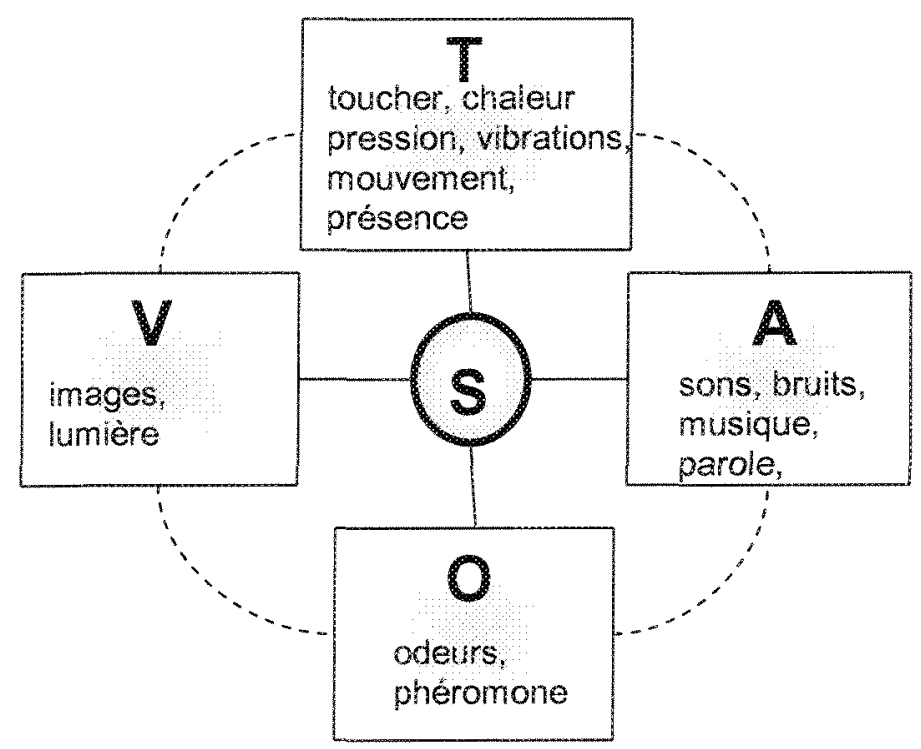

Figure 12. Stimuli à l'cuvre lors de l'évènement scénique. T : tactiles et kinesthésiques, $\mathrm{V}$ : visuels, $\mathrm{O}$ : olfactifs, $\mathrm{A}$ : auditifs, $\mathrm{S}:$ spectateur 
L'origine de ces signaux est variable et peut provenir autant du performer que de d'autres sources externes. Lors de l'évènement scénique, le spectateur n'est pas uniquement récepteur (même si c'est le principal intéressé), mais devient lui-même producteur de signaux (dans une moindre mesure cependant, mais l'échange, la circulation est réelle). Après avoir spécifié la nécessité d'un art performatif organique de la part du performer, l'important n'est pas de déterminer l'origine de ces signaux, car leurs sources sont multiples et complexes, mais de savoir comment ces signaux, ces stimuli, sont traités par le corps pour définir l'expérience sensible du spectateur.

\section{Systèmes de traitement}

Selon la forme matérielle que prend le signal, deux systèmes de régulation entrent en jeu lors de la communication inteme et externe du corps humain, donc également chez le spectateur d'art performatif: le système nerveux et le système endocrinien $^{34}$.

\footnotetext{
${ }^{34}$ Les informations scientifiques qui suivront sont tirées de: Campbell, N.A. (1995). Biologie. Canada: Éditions du Renouveau Pédagogique Inc.
} 


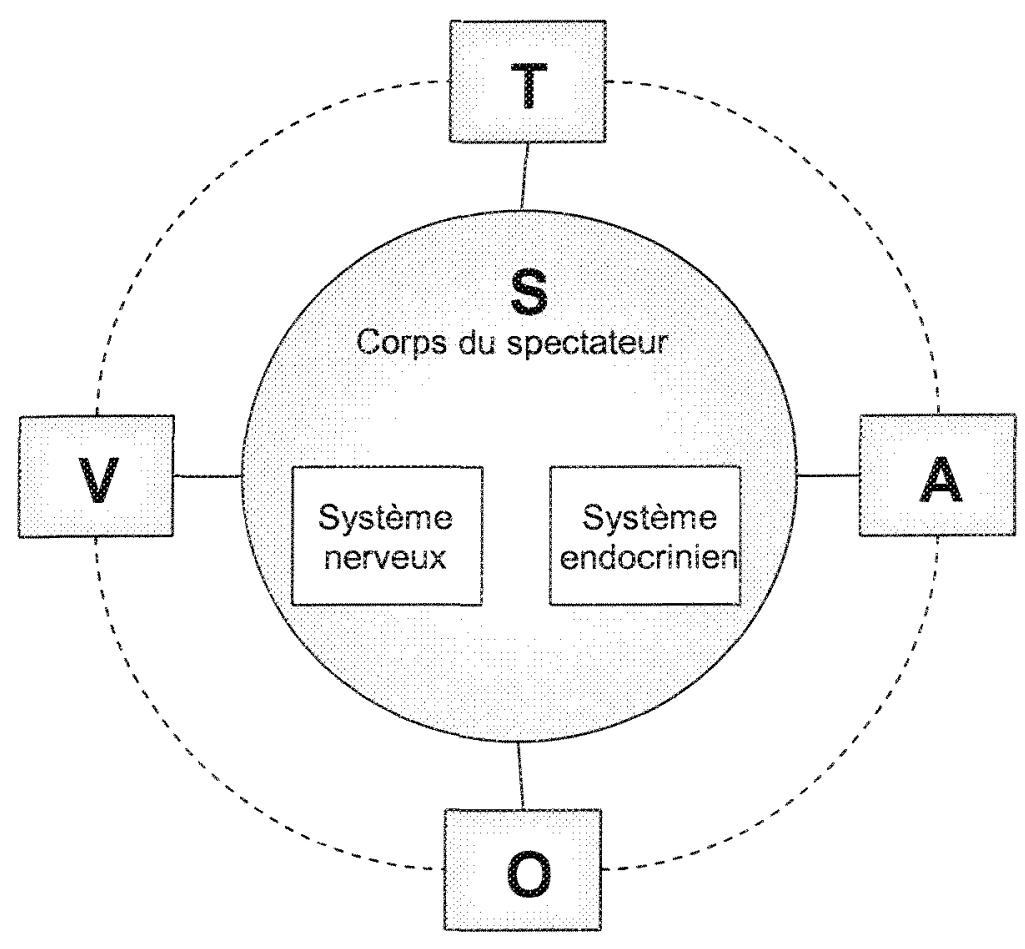

Figure 13. Systèmes de traitement des stimuli

Dans de nombreux cas, ces deux systèmes coopèrent et interagissent pour influer sur la physiologie et le comportement. Cependant, comme la structure du système endocrinien et celle du système nerveux diffèrent grandement, ces deux systèmes présentent d'importantes divergences sur le plan fonctionnel.

Le système endocrinien se compose de glandes individuelles, disséminées dans l'ensemble de l'organisme, qui envoient leurs messagers chimiques (hormones) par 
l'intermédiaire de la circulation sanguine à des cellules cibles dispersées. Ce système est principalement affecté à la régulation interne de l'organisme.

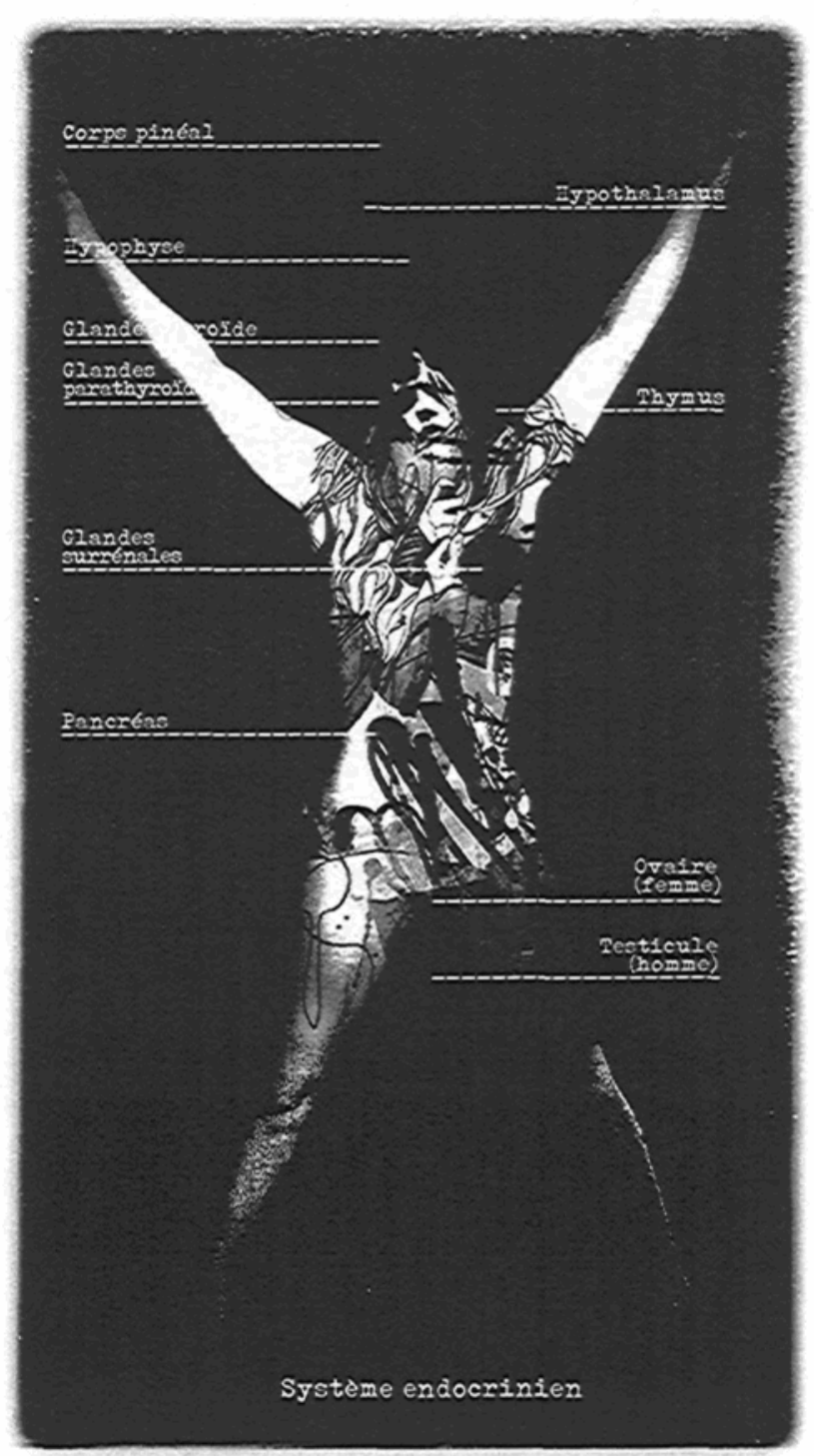

Figure 14. Système endocrinien.

En revanche, le système nerveux est un réseau de communication dont les ramifications acheminent directement l'information voulue, soit sous forme de 
commandes à des tissus cibles spécifiques situés dans diverses régions du corps, soit sous forme de messages qui en proviennent.

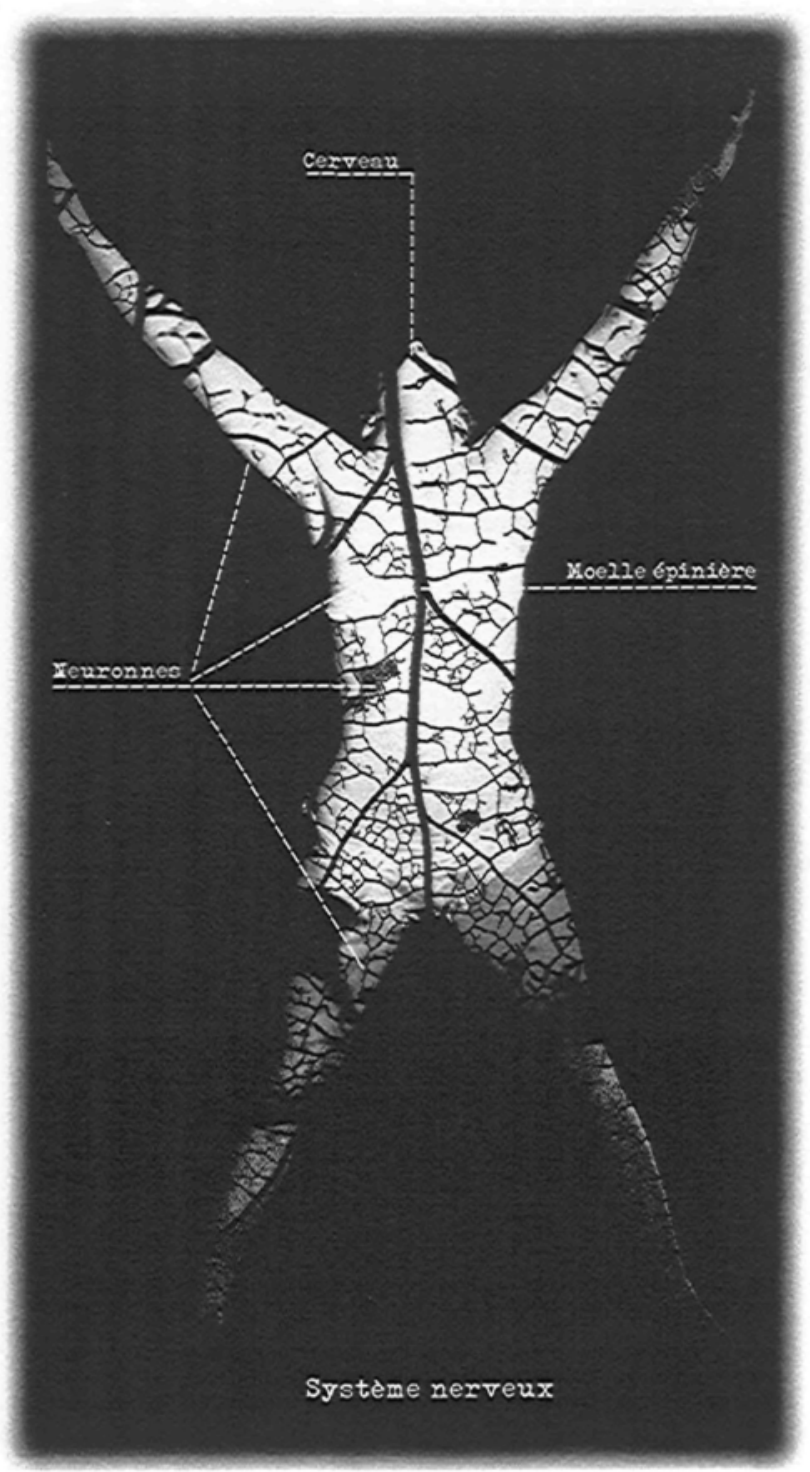

Figure 15. Système nerveux 
D'ailleurs, le système nerveux est probablement la structure la plus complexe sur la Terre. Dans à peine $1 \mathrm{~cm}^{3}$ de cerveau humain peuvent se trouver plusieurs millions de neurones, et chacun peut entrer en communication avec des milliers d'autres; le tout forme un réseau de traitement de l'information à côté duquel les circuits intégrés les plus perfectionnés jamais construits par l'humain paraissent bien primitifs. Ces voies nerveuses déterminent chacune de nos perceptions et chacun de nos mouvements et, d'une certaine façon, rendent possible l'apprentissage, la mémoire et la pensée.

Étant donné que les cellules du système nerveux (neurones) sont spécialisées dans la transmission rapide de messages par courants électriques, cette structure permet à l'information de voyager beaucoup plus rapidement que dans le système endocrinien, Les messages peuvent circuler le long des neurones à la vitesse de $100 \mathrm{~m} / \mathrm{s}(360 \mathrm{~km} / \mathrm{h})$, alors que cela prend habituellement plusieurs secondes ou minutes lorsqu'il s'agit du système endocrinien.

Le système nerveux se caractérise également par l'extrême précision de son fonctionnement. Les hormones libérées par une glande endocrine sont distribuées dans tout l'organisme par l'intermédiaire du système cardiovasculaire, alors que le système nerveux peut donner lieu à des mouvements très fins en stimulant la contraction de quelques muscles, voire d'un seul, à un instant donné. 
Une troisième différence est l'extrême complexité structurale du système nerveux, qui lui permet d'intégrer une foule d'informations diverses et de déclencher un plus vaste éventail de réponses que le système endocrinien.

De manière générale, le système nerveux possède trois fonctions qui se chevauchent: la réception d'informations sensorielles, l'intégration et l'émission de commandes motrices. Lors de l'évènement scénique, il y a, en quelque sorte, censure de cette troisième fonction. C'est-à-dire que le spectateur ne produit pas (ou très peu) de commandes motrices visuellement repérables par les autres. Cependant, cela ne veut surtout pas dire qu'il ne se passe rien dans le corps de ce dernier.

La première fonction permet d'acheminer les informations provenant des récepteurs sensoriels, comme par exemples les cellules photosensibles des yeux, vers les centres d'intégration du système nerveux.

L'intégration est le mécanisme d'interprétation de l'information émanant, d'une part, de la stimulation des récepteurs sensoriels par le milieu et, d'autre part, de la recherche d'une réponse adéquate par l'organisme. La plus grande partie de l'intégration s'effectue dans le système nerveux central (SNC), composé de l'encéphale et de la moelle épinière. 
Les commandes motrices partent du centre de traitement, c'est-à-dire du système nerveux central, et se rendent aux cellules effectrices, soit les cellules des muscles ou des glandes qui donnent véritablement la réaction de l'organisme aux stimuli. Les nerfs qui transmettent les messages moteurs et sensitifs entre le système nerveux central et le reste de l'organisme sont appelés système nerveux périphérique (SNP).

C'est en partie lors de cette troisième fonction, l'émission de commande motrice, que la magie opère au théâtre, ou plus précisément lors de l'évènement scénique. En effet, quand le spectateur reçoit et intègre l'information véhiculée par les signaux, il est rare qu'un mouvement apparent en résulte. Des changements de tension dans le corps de ce dernier, des micro-mouvements, des réactions physiologiques comme la sudation ou même des réactions émotives telles que le rire et les larmes surviennent assurément, mais rarement un mouvement, un geste repérable par les autres spectateurs présents.

La magie dont je parle provient en grande partie de la théorie des neurones miroirs de Rizzolatti. C'est-à-dire que le corps du spectateur est réellement stimulé par le mouvement du performer, ses propres schémas d'émission de commandes motrices, son chemin neuronal pour effectuer le même mouvement qu'il perçoit, est activé comme s'il allait le produire lui-même, sans que cela se passe. Mais, cette théorie sera développée plus loin. 
Pour en revenir à nos systèmes de traitement, l'information passe donc d'un neurone à l'autre, et cela du récepteur à l'effecteur, grâce à un ensemble de phénomènes électriques et chimiques.

\section{Nuance entre la sensation et la perceptions}

Afin de clarifier les termes employés, il importe de faire une distinction entre sensation et perception : la sensation résulte d'un processus physiologique immédiat lié à nos organes récepteurs de stimuli, tandis que la perception découle de l'interprétation de ces sensations par le cerveau.

Dans la présente recherche, la perception, comme nous venons de la décrire, n'est pas abordée. C'est la sensation qui est surtout traitée, d'où la justification de l'emploi du qualificatif « sensible» dans l'expérience du spectateur.

L'information circule dans le système nerveux sous forme de potentiel d'action. Un potentiel d'action généré par la lumière qui atteint l'œil est de même nature qu'un potentiel d'action créé dans l'oreille par les vibrations de l'air. Cependant, nous faisons facilement une distinction entre la stimulation lumineuse et la stimulation sonore. Cette distinction dépend de la région de l'encéphale qui reçoit le message. 
Par exemple, l'oreille convertit en influx nerveux les vibrations de l'air que nous appelons sons. Les neurones sensitifs acheminent ces influx, nommés sensations, jusqu'à une région précise du cortex cérébral, sous forme de potentiel d'action. Lorsque le cerveau prend conscience de ces sensations, il les interprète et nous procure la perception des sons. D'autres types d'information sont envoyés à d'autres régions de l'encéphale et donnent naissance à des perceptions différentes. Ce qui importe donc, c'est l'endroit où parvient l'influx et non ce qui l'a provoqué.

Les sensations et les perceptions qu'elles engendrent dans le cerveau trouvent leur origine dans l'excitation des récepteurs sensoriels, des structures qui transmettent des informations relatives aux modifications survenant dans les milieux internes et externes du corps.

Ces structures assurent une fonction précise, soit la réaction à divers stimuli, dont la chaleur, la lumière, la pression et les substances chimiques. Tous ces stimuli, les signaux développés précédemment, représentent des formes d'énergie. D'une façon générale la fonction des cellules réceptrices consiste à convertir l'énergie des stimuli en énergie électrochimique, c'est-à-dire en potentiel d'action, et à faire parvenir ces derniers au système nerveux. Ce processus se divise en cinq fonctions communes à toutes les cellules réceptrices: la réception, la conversion, l'amplification, la transmission et l'intégration. 


\section{Processus d'intégration de l'information sensorielle chez le spectateur}

Peu importe le terme employé, biosème, signal ou stimulus, voilà comment cette énergie qui circule lors de l'évènement scénique est traitée physiologiquement dans le corps du spectateur.

1- réception : capacité d'absorption de l'énergie représentée par un stimulus;

2- conversion : transformation de l'énergie du stimulus en activité électrochimique, autrement dit en influx nerveux;

3- amplification : l'énergie du stimulus s'avère souvent trop faible pour parvenir au système nerveux; elle doit subir une amplification;

4- transmission: l'énergie du stimulus, convertie en variation du potentiel de membrane, doit parvenir au système nerveux;

5- intégration : le traitement de l'information sensorielle se met en place dès sa réception; l'intégration des messages en provenance des récepteurs se réalise par sommation des potentiels gradués.

Afin que ce processus se produise, plusieurs récepteurs sont mis en jeu lors de l'expérience sensible. On divise les récepteurs sensoriels en deux grands groupes : les extérocepteurs, qui reçoivent l'information en provenance du milieu extérieur (lumière, son, toucher), et les intérocepteurs, qui foumissent des renseignements sur le milieu interne. Les récepteurs sont également classés en fonction du type d'énergie que 
représente la stimulation: les mécanorécepteurs, les chimiorécepteurs, les récepteurs d'ondes électromagnétique, les thermorécepteurs, et les nocicepteurs (récepteurs de la douleur).

Mécanorécepteurs : ces récepteurs réagissent à des déformations physiques dues à des phénomènes tels que la pression, le toucher, l'étirement, le mouvement et le son; ces phénomènes représentent tous des formes d'énergie mécanique. Voici quelques exemples:

- Corpuscules de Ruffini : se trouvent sous l'épiderme de la peau; détectent la pression intense et les étirements.

- Corpuscules lamelleux (corpuscules de Pacini): se trouvent dans les couches profondes de la peau; réagissent rapidement aux pressions intenses.

- Corpuscules tactiles capsulés (corpuscules de Meissner): se trouvent près de la surface de la peau; réagissent à la pression légère, les vibrations et le toucher discriminant.

- Corpuscules bulboïdes (corpuscules de Krause) : se trouvent près de la surface de la peau; détectent la pression légère.

- Corpuscules tactiles non capsulés: se trouvent près de la surface de la peau; détectent les contacts légers.

- Fuseau neuromusculaire : ce récepteur de tension est un exemple d'intérocepteurs qui perçoit la longueur des muscles squelettiques.

- Cellule sensorielle ciliée : sert à détecter le mouvement, sa force et sa vitesse. Joue également un rôle dans l'audition et l'équilibre; se retrouve dans l'oreille. 


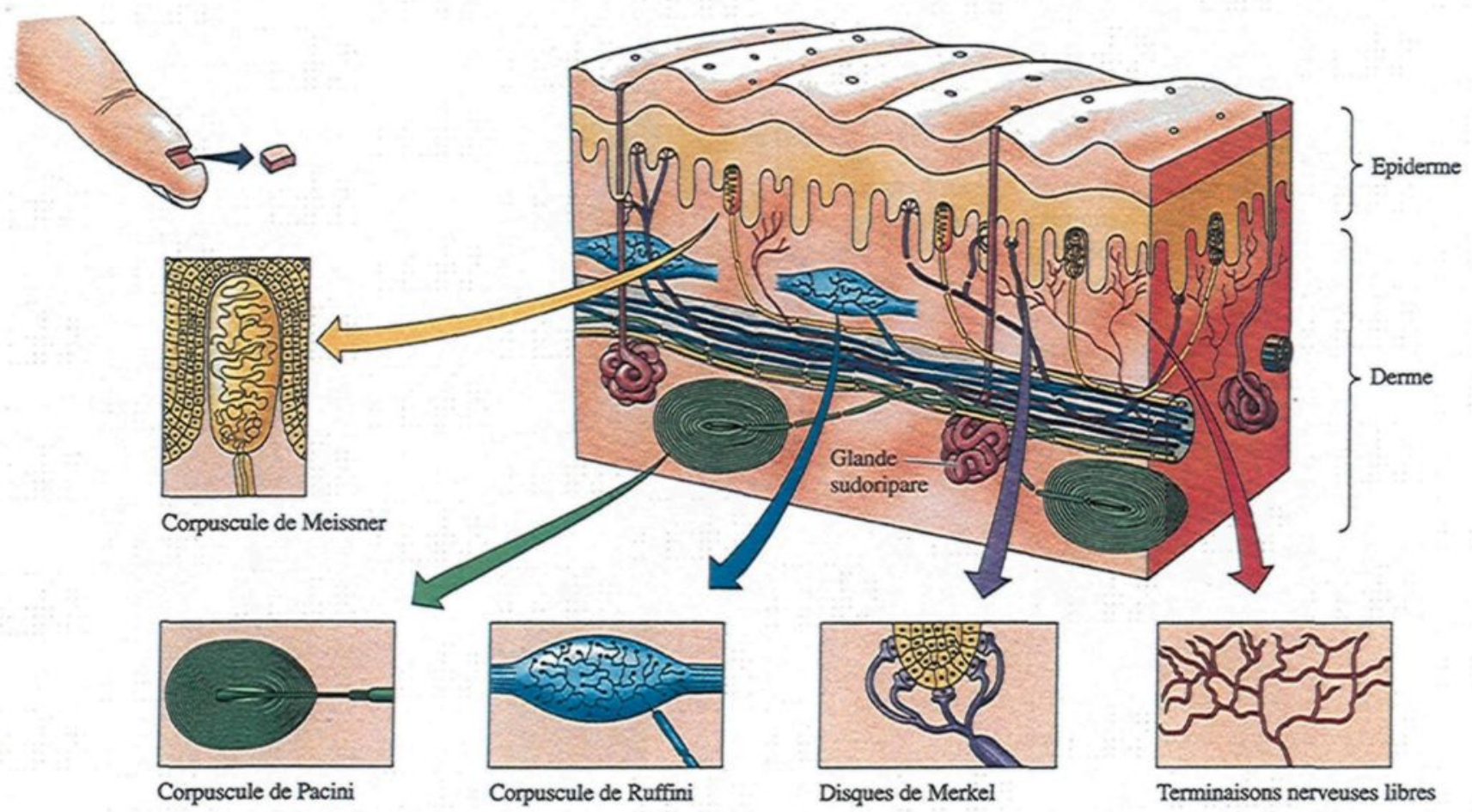

Figure 16. Quelques uns des récepteurs de la peau

Récepteurs d'ondes électromagnétiques : les rayonnements électromagnétiques sont une forme d'énergie pouvant correspondre à différentes longueurs d'onde; la lumière visible, l'électricité et le magnétisme en constituent les manifestations.

- Photorécepteurs : ils détectent le rayonnement que nous appelons lumière visible; ces récepteurs se situent le plus souvent dans les yeux.

Chimiorécepteurs, thermorécepteurs et récepteur de la douleur: ces récepteurs ne seront pas décrits ici puisqu'ils ne servent pas mon propos. 


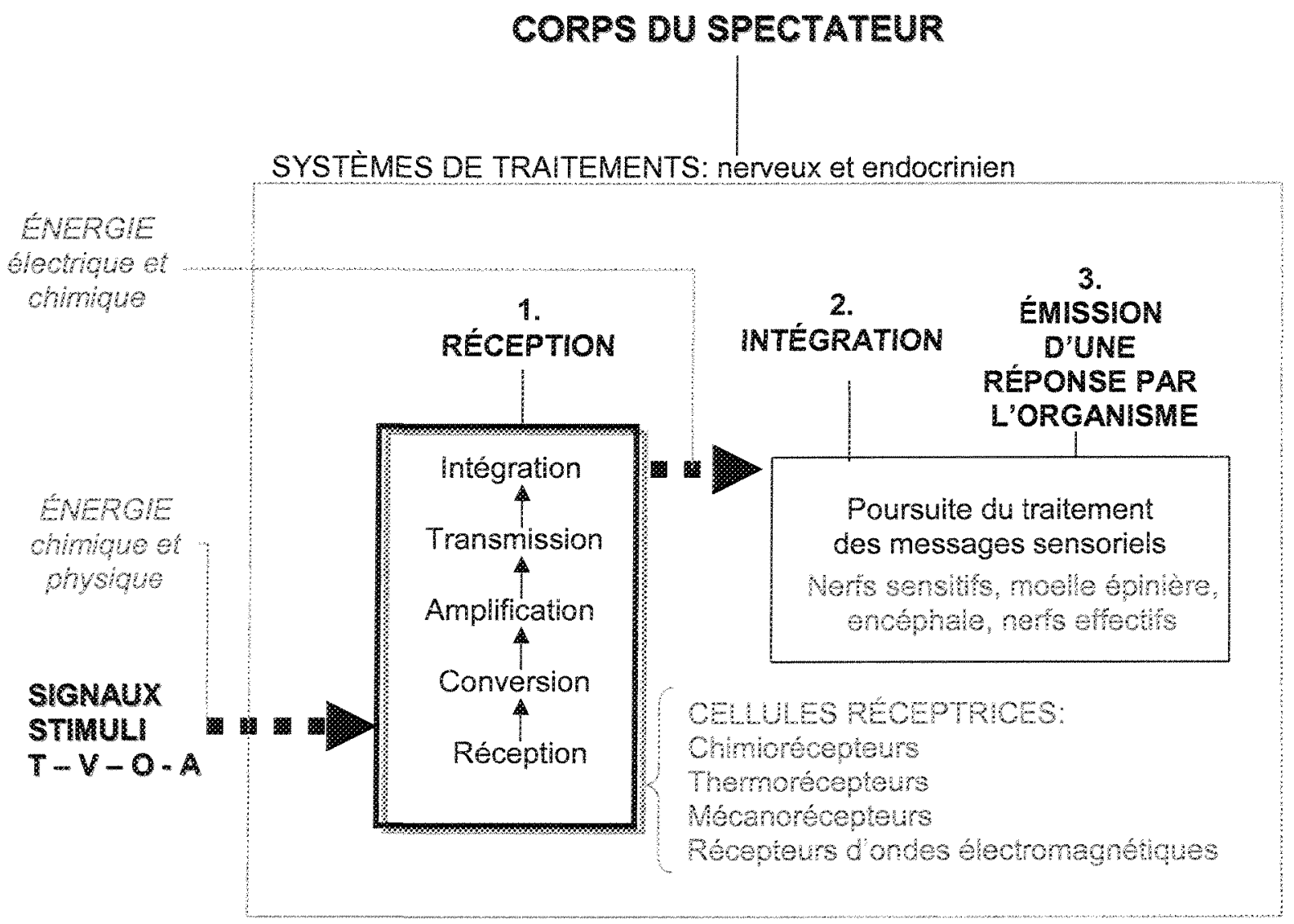

Figure 17. Physiologie de l'expérience sensible

\section{L'empathie physiologique}

Cette partie de la recherche trace un pont entre le vaste univers scientifique et mes intuitions théâtrales: 
Les arts scéniques, en invitant à une exposition corporelle directe entre les participants, sollicitent intensément les processus d'empathie psycho-biologique, qui reposent sur des signaux non-verbaux, sur des mobilisations physiologiques intenses et subtiles. Cetie communication empathique faite de simulations neuronales, de contagions tonico-rythmiques et de résonances cénesthésiques, nous semble être un élément essentiel de l'échange scénique. ${ }^{35}$

\section{Les origines}

Le concept d'empathie remonte à plusieurs décennies. Selon Gérard Jorland, l'empathie serait née avec Adam Smith au milieu du $18^{\text {e }}$ siècle. Depuis, le concept a migré sans cesse à travers toutes les disciplines, que ce soit de la morale à l'esthétique, en passant par lhistoire naturelle, la psychologie, la philosophie et même la théologie. En 1872, Robert Visher introduit le terme Einfiihlung, mais c'est Theodor Lipps qui fait passer le concept en psychologie, l'élabore et en fait finalement le fondement de l'esthétique.

Dans un autre ordre d'idées, l'émergence de l'imagerie mentale et de la technologie qui «laisse voir le cerveau penser» (ex : résonance magnétique, scanographie), a redonné une nouvelle forme de vie ainsi qu'un nouvel intérêt à l'étude de tous les processus mentaux, dont l'empathie.

\footnotetext{
${ }^{35}$ Leão, M. (2003). La présence totale au mouvement. France : Éditions Point d’Appui, page 63.
} 
La définition du phénomène d'empathie se fond à la discipline d'où il émerge. C'est un concept passe-partout. Cependant, celle que j'ai choisie tentera de mettre l'accent sur la physiologie, avec la théorie des neurones miroirs, afin de se rapprocher le plus possible de ma recherche. Il est tout de même important de souligner que l'empathie se compose, en grande partie, de processus cognitifs et affectifs, superposés à une forme de résonance motrice «dont le déclenchement est le plus souvent automatique, non-contrôlable, et non intentionnel ${ }^{36}$.

C'est avant tout une forme d'intersubjectivité, un des nombreux canaux que peut emprunter le contact d'altérité. Cela suppose une capacité à se mettre à la place de l'autre, la plupart du temps de façon intuitive et non-consciente, à partager, à comprendre ses états physiques et mentaux. La mise en présence de deux organismes vivants, lors de l'évènement scénique, dessine un contexte, une situation presque idéale afin que l'empathie résonne.

L'empathie est même qualifiée de «forme de communication spontanée, préconceptuelle, dont on sait aujourd'hui qu'elle est extrêmement précoce: les observations de Meltzoff et al. (1977; 1983) mettent en évidence que des nouveau-nés de 32 heures présentent des conduites imitatives. ${ }^{37}$. Ce qui veut dire que l'humain, presque dès sa naissance et aussi immature soit-il, est un partenaire de communication à part entière en ce sens qu'il émet un signal et attend une réponse.

\footnotetext{
${ }^{36}$ Berthoz, A., \& Jorland, G. (2004). L'empathie. Paris : Editions Odile Jacob.

37 Ibid.
} 
Jean Decety précise que le phénomène d'empathie est une capacité propre à la nature humaine, qui repose sur des systèmes neurologiques que l'on commence à comprendre. Son idée principale se résume ainsi : l'empathie est une simulation mentale de la subjectivité d'autrui. Toujours selon lui, la caractéristique essentielle de l'empathie réside dans la distanciation entre soi et autrui, parallèlement avec un partage affectif. II est primordial de faire une distinction consciente que l'autre nous est semblable, mais sans confusion avec nous-mêmes et lui: "l'empathie implique un mécanisme de résonance avec les affects inconscients de l'autre sans que la personne ne perde son intégrité. $\rangle^{38}$.

Alain Berthoz ajoute que l'empathie nécessite un changement de référentiel, un mécanisme cognitif essentiel pour vivre l'empathie. C'est-à-dire qu'au lieu de saisir la réalité avec notre habituel référentiel égocentré, il faut passer par le référentiel hétérocentré, même allocentré; en simplifiant l'interprétation: avoir un point de vue centré sur l'autre. "C'est un double vécu dont il s'agit, curieux mélange de soi et l'autre ${ }^{39}$, dit-i1. Il insiste en affimant que le secret de l'empathie ne se trouve pas seulement dans les neurones miroirs, ni dans la capacité de simuler mentalement les actions de l'autre ou d'en éprouver les émotions, « il exige cette capacité de changer de point de vie tout en gardant le sentiment de soi. $" 40$.

\footnotetext{
${ }^{38}$ Ibid.

${ }^{39}$ Ibid.

${ }^{40}$ Ibid
} 
La théorie empathique de Berthoz nous mène à celle du cerveau projectif. $\mathrm{Ce}$ chercheur soutient, comme plusieurs scientifiques contemporains, que notre cerveau est un simulateur du monde extérieur, «qu'il projette sur le monde ses règles d'analyses, ses préperceptions, c'est un générateur d'hypothèses (...), le cerveau est prêt à déformer le monde en fonction de ses règles internes. " ${ }^{4}$. Berthoz affirme alors que l'empathie est une propriété fondamentale du cerveau percevant plutôt qu'une simple théorie superposée à son fonctionnement. En effet, il spécifie que le cerveau qui perçoit est toujours en train de projeter sur le monde ses propres lois; percevoir c'est identifier le monde et le sujet agissant.

L'évènement scénique, qui invite à une présence corporelle directe entre le performer et le spectateur, sollicite le phénomène d'empathie dans son ensemble. Ce phénomène confirme l'immense subjectivité de chaque spectateur liée à sa propre expérience. La mémoire affective de ce dernier, les souvenirs, la culture, l'identité, l'état émotif et physiologique de l'instant sont tous autant de facteurs déterminants des mécanismes cognitifs, affectifs et physiologiques de l'expérience sensible du spectateur. Ils teintent le changement de référentiel du spectateur, son échange empathique avec le performer, parce qu'assurément l'empathie est présente dans cet échange artistique et physiologique.

41 Ibid 


\section{La théorie des neurones miroirs}

Cette théorie me fait revenir à l'aspect « physiologique » de l'empathie, le tronc commun avec l'expérience sensible du spectateur. La théorie des neurones miroirs a été découverte par le professeur Giacomo Rizzolatii ${ }^{42}$ et son équipe de l'université de Parme, il y a environ dix ans.

En étudiant le système moteur chez le singe, cette équipe a découvert que les neurones miroirs s'activaient non seulement lorsque le singe effectuait une certaine action, mais également lorsqu'il observait quelqu'un exécuter cette même action. Les études se sont poursuivies chez l'homme et d'autres chercheurs, comme Tania Singer, ont confirmé l'existence de ces neurones miroirs dans les zones morrices, mais également dans les zones émotionnelles. Une expérience menée sur une femme a démontrée que les mêmes zones s'activaient lorsqu'elle éprouvait de la douleur, et quand elle voyait la douleur chez l'autre: «Ceci signifie que lorsque nous voyons un autre souffrir, c'est comme si nous souffrions nous-mêmes. $»^{43}$.

Avec la théorie des neurones miroirs, nous découvrons que l'empathie a réellement une implication physiologique et que cette capacité est innée chez l'homme. Les expériences menées chez les nouveau-nés le prouvent.

\footnotetext{
${ }^{42}$ Science et conscience. (2009), page 22.

${ }^{43} \mathrm{Ibid}$
} 
Cette réelle résonance physiologique devient très intéressante chez le spectateur d'évènement scénique. En effet, cette théorie, ici très simplifiée et vulgarisée, implique toute une série de réactions en chaîne dans le système nerveux lorsque l'on voit quelqu'un faire ou ressentir quelque chose. Le professeur Fogassi continue le raisonnement en nous révélant que ce mécanisme miroir nous donne accès à l'intentionnalité de l'autre: «Dans un geste, il y a toujours deux composantes, le comment et le pourquoi. Lorsque nous regardons un autre faire, nous comprenons vite ce qui se passe et en général, pourquoi (il agit ainsi). $>{ }^{44}$.

Le vieux concept de catharsis serait-il ici renouvelé par celui de l'empathie? Purgation d'idées, de fantasmes, de désirs (d'une façon réelle parce que tout notre corps s'active et résonne de façon miroir, comme si nous accomplissions ce que nous voyons, sans que nous bougions, sans que nous fassions aboutir le geste au final) en restant observateur de l'évènement scénique, sans en subir les conséquences?

Aristote parlait de la catharsis comme d'une «purgation» de l'affect à l'aide de l'identification au héros sur scène. Ce «double vécu, curieux mélange de soi et de l'autre» dont parle Berthoz, ne ressemble-t-il pas un peu à cette forme d'identification cathartique?

\footnotetext{
44 ibid
} 
Si la catharsis n'est pas renouvelée, parce que cela demeure un concept assez complexe, doté de multiples explications qui ne s'accordent pas toujours, elle est assurément complétée par le concept d'empathie. Cet aspect physiologique de l'expérience sensible chez le spectateur foumit, pour moi, un éclairage prometteur sur ce phénomène esthétique lié au plaisir du spectateur.

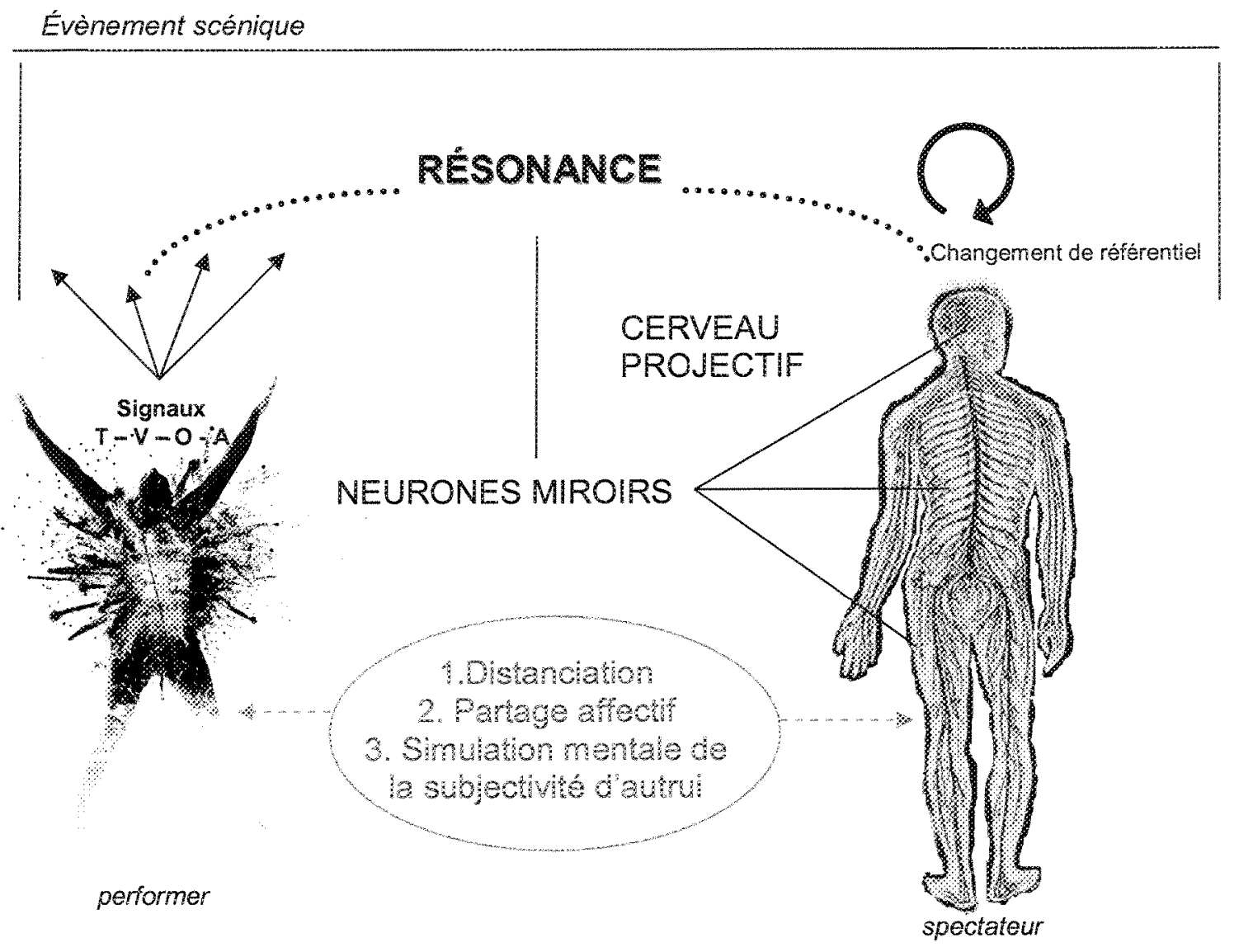

Figure 18. L'empathie physiologique 
CONCLUSION 
Je la perçois. Je sens ses os craquer jusqu'à moi, ses muscles crient, sa peau se déchire, son silence parle fort. Les molécules vibrantes me font résonner ses mouvements; la chaleur émane de son corps, active mes propres atomes, ma substance. Il y a le vide entre nous, un vide quantique. Il y a l'énergie, un plein processus qui s'agite sans cesse en signal. ll y a une masse humaine autour. Ils sont présents. Mes sens s'excitent, deviennent gradients de mon expérience.

J'écoute. J'écoute un texte, des phrases qui se suivent, des mots qui signifient. Mais tout à coup, je n'entends plus une histoire. Je ressens mon histoire. J'entends les sons qui étincellent et $m$ 'interpellent. Ces ondes deviennent la musique de ma propre vie. Mon corps intègre ces vibrations. Elles résonnent jusqu'au tréfonds de ma mémoire, de mes désirs. L'expérience prend vie, se matérialise dans ma chair pensante, infusée de toute part par mes neurones.

Je ressens. Sublimation de mes désirs noyés dans mes sowvenirs, qui tracent leur sillon au creux des circonvolutions de mon écorce cérébrale. L'encéphale est mémoire affective. Cognitivo-émotive. Physio-moléculaire. Nucléo-génétique.

Effectuer une incursion physiologique au niveau de la perception dans le corps du spectateur, dans l'expérience sensible qui fonde le paysage vivant de l'évènement scénique: mon ambition se résumait ainsi. Je me suis donc accrochée à l'évènement d'art performatif comme à un processus biologique, assimilable à d'autres réactions scientifiques. En fait, c'est ainsi que je le perçois, dans mon obsession systémique, ma fascination pour «l'univers système » et ses embôttements infinis.

Les niveaux d'organisation multiples de l'expérience sensible ont donc été traités à l'aide de l'approche systémique, c'est-à-dire par une modélisation de la complexité que l'on tente de saisir. Avoir une vision globale de ce phénomène d'art performatif m'a 
permis de le relier au concept d'empathie physiologique, de manière cohérente, me semble-t-il.

L'ethnoscénologie s'est présentée comme un cadre pour cemer mes réflexions sur un théâtre biologique. Les théories sur cet aspect vivant de l'évènement scénique sont rares. Mes recherches de réponses physiologiques à ce questionnement esthétique m'ont fait atterrir dans l'univers fascinant des neurosciences. C'est à partir de là que j'ai tenté de mettre en lien la théorie des neurones miroirs, le principal aspect du concept d'empathie physiologique, avec l'évènement scénique.

Par contre, pour que cette liaison fonctionne, je crois que l'évènement théâtral doit appartenir à une forme performative d'art, une mise en présence qui stimule véritablement l'organisme du spectateur. L'échange se doit d'être organique pour qu'il se produise une contagion kinesthésique, pour que le phénomène d'empathie physiologique résonne dans la matrice corporelle du récepteur en activant ses neurones miroirs.

Cette résonance s'effectue de façon tangible grâce aux signaux qui circulent lors de l'évènement scénique. Cette forme d'énergie qu'est le signal établit la communication entre l'évènement scénique (surtout le performer) et le spectateur. Le signal est palpable, mesurable. Ces multiples vecteurs d'ordre chimique et/ou physique possèdent une intensité variable et, selon moi, l'expérience sensible du spectateur est 
directement proportionnelle à l'organicité du performer. Ces stimuli organiques sont, en quelque sorte, des catalyseurs positifs de l'expérience sensible.

Même si l'expérience, dans ce qu'elle a de plus total, est le résultat d'un incessant va-et-vient entre signification (sens) et signal, je pense que c'est au spectateur qu'appartient la construction principale du sens. Ce sont les signaux, les stimuli, dans ce qu'ils ont de plus matériel, qui sont les précurseurs de cette expérience sensible. Je m'explique. Chaque spectateur possède un sens commun, mais surtout une subjectivité qui lui est propre. C'est donc chacun, en fonction d'une foule de facteurs (origine, état, expérience, connaissances, mémoire affective, etc.) qui produit une interprétation unique, donc du sens, grâce à l'intégration première par le système nerveux et endocrinien de ces signaux, ultimement remaniés par le cerveau projectif.

Bien sûr, chaque auteur, chaque metteur en scène, veut faire sens dans son œuvre. Cependant, ce sont les signaux émergeants qui ont le plus d'impact, de résonance sur le spectateur. Trop vouloir faire du sens, en usant d'une esthétique de la simulation par exemple, court-circuite l'aspect physiologique en faisant appel directement au raisonnement intellectuel du spectateur, donc à son interprétation. Cela m'amène à considérer le signal comme la seule véritable substance transigeante mise en cause lors de l'évènement scénique, entre le performer et le spectateur. Pascal Bouchez ajoute: 
«Nous sommes tous fort heureusement des êtres «résonateurs» dans toutes les strates et niveaux de notre corps avant d'être des êtres «raisonneurs»" ${ }^{45}$.

Voilà quelques-unes des conclusions à tirer de cette recherche. Par ailleurs, mesurant mieux combien cet aspect physiologique de l'évènement scénique joue un rôle fondamental dans l'activité spectatorielle, je sais que cette réflexion va transformer ma pratique personnelle. Bien entendu, il ne s'agit là que d'une partie de la réponse à la question de l'expérience sensible. Celle-ci est complexe et possède plusieurs niveaux qui s'entrecoupent. Il faudrait beaucoup plus de connaissances pour en saisir toutes les subtilités d'ordre physiologique, oui, mais aussi cognitif, émotionnel et neurologique.

J'ai tout de même l'impression que cette amorce résonne comme un écho de la « crise » du théâtre actuel, qui, telle que rapportée par la théorie du postdramatique, met davantage en cause la substance biologique de cet art de relation qu'est le théâtre. Ce que Jean-Marie Pradier démontre d'ailleurs avec force dans son manifeste sur l'ethnoscénologie.

Cette recherche ne fait que commencer. Etre capable de vraiment fouiller et décortiquer l'aspect neurologique de l'expérience sensible au théâtre amènerait de nouvelles réponses, sûrement passionnantes. Par contre, malgré l'évolution fulgurante des neurosciences, on commence à peine à superposer avec certitude un aspect

${ }^{45}$ Bouchez, P. (2007). Filmer l'éphémère : récrire le théâtre (et Mesguich) en images et en sons. Paris : Presses Universitaires du Septentrion. p39 
physiologique aux facultés supérieures de l'homme, telles la conscience, la mémoire et le plaisir esthétique. Le cerveau et ses milliards de connexions synaptiques gardent toujours une bonne partie de ses mystères.

Le développement de ces neurosciences est également la preuve de la fin du clivage cartésien entre le corps et l'esprit. Les facultés «supérieures» sont désormais soumises à une vision systémique de la complexité et des niveaux d'organisation du système nerveux, ainsi que de l'encéphale. La multiplicité des interconnexions entre nos neurones, dont la majeure partie s'établit en très bas âge, module notre compréhension, mais avant tout notre expérience sensible, de la vie dans sa totalité.

J'écris ces lignes et je regarde ma petite fille de deux ans. Cet être complètement empathique, qui se développe à une vitesse folle, éponge physiologiquement tous les instants de la vie pour modeler ses interconnexions, construire son propre empire de sens. C'est à ce moment précis, loin du théâtre, dans la profondeur de ses yeux, que je redécouvre la pertinence et l'intérêt de ma recherche. 


\section{Société}
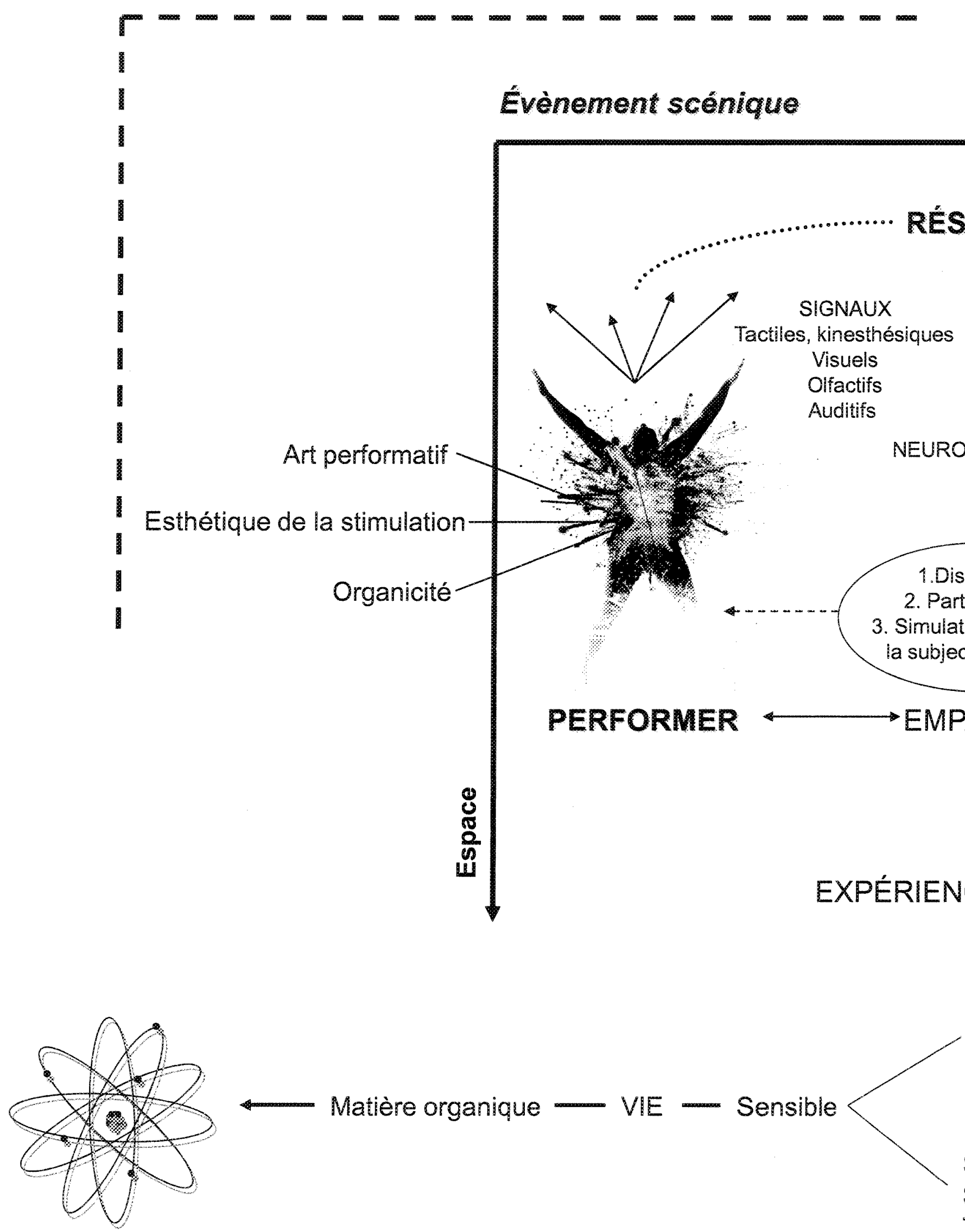

$\longleftarrow$ Matière organique - VIE - Sensible 